\title{
FORTY-EIGHTH
}

\section{Asilomar Conference on}

\section{Signals, Systems and}

Computers

Final Program

November 2-5, 2014

Asilomar Hotel and

Conference Grounds

Technical Co-sponsor

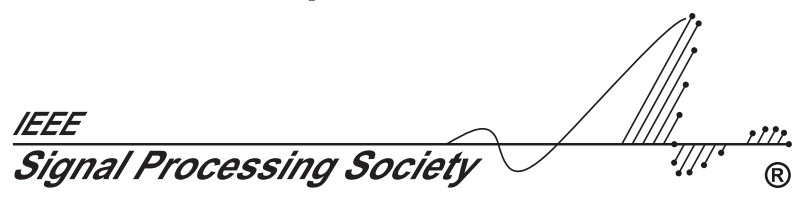




\title{
FORTY-EIGHTH ASILOMAR CONFERENCE ON SIGNALS, SYSTEMS \& COMPUTERS
}

\author{
Technical Co-sponsor
}

IEEe Signal Processing Society

\section{CONFERENCE COMMITTEE}

\section{General Chair}

Prof. Roger Woods

School of Electronics, Electrical

Engineering and Computer

Science

Queen's University Belfast

Queen's Road, Queen's Island

Belfast, BT3 9DT, UK

\section{Technical Program Chair}

Prof. Geert Leus

Delft University of Technology

Fac. of Electrical Engineering, Mathematics and Computer Science

Mekelweg 4

2628CD Delft, The Netherlands

\section{Conference Coordinator}

Monique P. Fargues

Department of Electrical \&

Computer Engineering

Naval Postgraduate School

Monterey, CA 93943

E-mail: fargues@asilomarssc.org

\section{Publication Chair}

Michael Matthews

ATK Space Systems

10 Ragsdale Drive, Suite 201

Monterey, CA 93940

E-mail:

michael.matthews@ atk.com
Publicity Chair

Linda S. DeBrunner

Department of Electrical \& Computer Engineering

Florida State University

Tallahassee, FL 32310-6046

E-mail:

Linda.debrunner@eng.fsu.edu

\section{Finance Chair}

Ric Romero

Department of Electrical \& Computer Engineering

Naval Postgraduate School

Monterey, CA 93943-5121

E-mail: treasurer@ asilomarssc.org

Electronic Media Chair

Prof. Marios S. Pattichis

Department of Electrical \&

Computer Engineering

MSC01 1100

1 University of New Mexico

ECE Bldg., Room 125

Albuquerque, NM 87131-0001

Student Paper Contest Chair

Prof. Joseph R. Cavallaro

Rice University

Dept. of Electrical and Computer

Engineering

6100 Main Street, MS 380

Houston, TX 77005 


\title{
Welcome from the General Chairman
}

\author{
Prof. Roger Woods \\ Queen's University Belfast, UK
}

Welcome to the 48th Asilomar Conference on Signals, Systems, and Computers! I have had a long involvement with the Conference since my first publication in 1997 when I was immediately struck by the unique nature of the Asilomar conference environment. The picturesque sand dunes and warm sunshine provide a wonderful backdrop to a conference that allows easy access to, and interaction with key researchers. Understandably, over the years, I have needed little persuasion to attend. There will never be a better opportunity to capture the attention of a key researcher in your area of expertise than at Asilomar!

The technical program was crafted expertly by the Technical Program Chair, Geert Leus, and his team of Technical Area Chairs: Shengli Zhou, Zhengdao Wang, Bhaskar Rao, Michael Rabbat, Zhi Tian, Visa Koivunen, Selin Aviyente, Jorn Janneck, Mohsin Jamali, and Matt McKay. I would like to thank Geert and his team for assembling a high quality program with 437 accepted papers and 163 invited papers. The student paper contest this year has been chaired by Joe Cavallaro and he has selected a total of 11 submissions. The student finalists will present poster presentations to the judges on Sunday afternoon and, of course, everyone is welcome to attend. The awards for the top three papers will be made at the plenary session. A key innovation this year has been to inculcate two major themes, brain machine interface and neural networks, and processing of high dimensional large scale data.

This year's plenary talk will be given by Professor Georgios B. Giannakis, from the University of Minnesota. I am pleased to have such a high profile speaker with a strong background in signal processing across a wide range of applications. Georgios will describe signal processing techniques to handle massive datasets which are noisy, incomplete, vulnerable to cyber-attacks and have outliers. The growth of Big Data represents a major ongoing challenge for humanity. The derivation of suitable data processing techniques is a vital activity and I am especially looking forward to seeing what can be accomplished in this area. Georgios has had a long engagement with the conference having acted as part of the technical committee as early as 1993 and presented his first paper at Asilomar in 1988.

I am privileged to have served as this year's General Chair. I hope that you enjoy the 2014 Conference programme whilst taking some time out to encounter the very special environment and atmosphere that Asilomar has to offer.

Prof. Roger Woods

Queen's University Belfast, UK, June 2014 


\section{Conference Steering Committee}

\section{PROF. MONIQUE P. FARGUES}

President \& Chair

Electrical \& Computer Eng. Dept. Code $\mathrm{EC} / \mathrm{Fa}$

Naval Postgraduate School

Monterey, CA 93943-5121

fargues@asilomarssc.org

\section{PROF. SHERIF MICHAEL}

Secretary

Electrical \& Computer Eng. Dept. Code EC/Mi

Naval Postgraduate School

Monterey, CA 93943-5121

michael@nps.edu

\section{PROF. RIC ROMERO}

Treasurer

Electrical \& Computer Eng. Dept.

Code EC/Rr

Naval Postgraduate School

Monterey, CA 93943-5121

treasurer@asilomarssc.org

\section{PROF. SCOTT ACTON}

Electrical \& Computer Eng. Dept.

University of Virginia

P.O. Box 400743

Charlottesville, VA 22904-4743

acton@ virginia.edu

\section{PROF. MAITE BRANDT-PEARCE}

Electrical \& Computer Eng. Dept.

University of Virginia

P.O. Box 400743

Charlottesville, VA 22904

mb-p@virginia.edu

\section{PROF. LINDA DEBRUNNER}

Publicity Chair

Electrical \& Computer Eng. Dept.

Florida State University

2525 Pottsdamer Street, Room A-341-A

Tallahassee, FL 32310-6046

linda.debrunner@eng.fsu.edu

\section{PROF. VICTOR DEBRUNNER}

Electrical \& Computer Eng. Dept.

Florida State University

2525 Pottsdamer Street, Room A-341-A

Tallahassee, FL 32310-6046

victor.debrunner@eng.fsu.edu

\section{PROF. MILOS ERCEGOVAC}

Computer Science Dept.

University of California at Los Angeles

Los Angeles, CA 90095

\section{PROF. BENJAMIN FRIEDLANDER}

Computer Eng. Dept.

University of California

1156 High Street, MS:SOE2

Santa Cruz, CA 95064

Benjamin.friedlander@gmail.com

PROF. fredric j. harris

Electrical Eng. Dept.

San Diego State University

San Diego, CA 92182

fred.harris@sdsu.edu

DR. RALPH D. HIPPENSTIEL

San Diego, CA 92126

rhippenstiel@yahoo.com

\section{PROF. W. KENNETH JENKINS}

Electrical Eng. Dept.

The Pennsylvania State University 209C Electrical Engineering West University Park, PA 16802-2705 jenkins@engr.psu.edu

\section{PROF. FRANK KRAGH}

Electrical \& Computer Eng. Dept. Code EC/Kr

Naval Postgraduate School Monterey, CA 93943-5121

frank.kragh@ieee.org

\section{DR. MICHAEL B. MATTHEWS}

Publications Chair

ATK Space Systems

10 Ragsdale Drive, Suite 201

Monterey, CA 93940

Michael.matthews@atk.com

\section{DR. MARIOS PATTICHIS}

Electrical \& Computer Eng. Dept. MSC01 1100

1 University of New Mexico ECE Bldg., Room: 229A

Albuquerque, NM 87131-000

Pattichis@ece.unm.edu

\section{PROF. JAMES A. RITCEY}

Electrical Eng. Dept.

Box 352500

University of Washington

Seattle, Washington 98195

ritcey@ee.washington.edu

\section{DR. MICHAEL SCHULTE}

AMD

11400 Cherisse Dr.

Austin, TX 78739

Michael.schulte@amd.com

PROF. EARL E. SWARTZLANDER, JR.

Electrical \& Computer Eng. Dept.

University of Texas at Austin

Austin, TX 78712

eswartzla@aol.com

\section{PROF. KEITH A. TEAGUE}

School Electrical \& Computer

Engineering / 202ES

Oklahoma State University

Stillwater, OK 74078

Keith.teague@okstate.edu

\section{DR. MILOŠ DOROSLOVAČKI}

General Program Chair (ex officio) Year 2012

Electrical and Computer Engineering Dept. George Washington University

Washington, DC doroslov@gwu.edu

\section{PROF. ROBERT HEATH}

General Program Chair (ex officio) Year 2013

Electrical \& Computer Eng. Dept. The University of Texas at Austin

Austin, TX 78712

rheath@ece.utexas.edu 


\title{
2014 Asilomar Technical Program Committee
}

\author{
Technical Chair \\ Prof. Geert Leus \\ Delft University of Technology
}

\section{Asilomar Technical Program Committee Members}

\author{
A: COMMUNICATIONS \\ SYSTEMS \\ Prof. Shengli Zhou \\ University of Connecticut \\ Prof. Zhengdao Wang \\ lowa State University \\ E: ARRAY SIGNAL \\ PROCESSING \\ Prof. Visa Koivunen \\ Aalto University \\ F: BIOMEDICAL SIGNAL AND \\ IMAGE PROCESSING \\ Prof. Selin Aviyente \\ B: MIMO COMMUNICATIONS \\ AND SIGNAL PROCESSING \\ Prof. Bhaskar Rao \\ University of California San Diego \\ C: NETWORKS \\ Prof. Michael Rabbat \\ McGill University \\ Michigan State University \\ G: ARCHITECTURE AND \\ IMPLEMENTATION \\ Prof. Jörn W. Janneck \\ Lund University \\ H: SPEECH \\ Image and Video Processing \\ D: SIGNAL PROCESSING AND \\ ADAPTIVE SYSTEMS \\ Prof. Zhi (Gerry) Tian \\ Michigan Technological University \\ Prof. Mohsin M. Jamali \\ University of Toledo \\ VICE CHAIR \\ Prof. Matthew McKay \\ Hong Kong University of Science \\ and Technology
}




\title{
2014 Asilomar Conference Session Schedule
}

\author{
Sunday Afternoon, November 2, 2014
}

$\begin{array}{ll}\text { 3:00-7:00 PM } & \text { Registration - Merrill Hall } \\ \text { 4:00-6:30 PM } & \text { Student Paper Contest - Heather } \\ \text { 7:00-9:00 PM } & \text { Welcoming Dessert Reception — Merrill Hall }\end{array}$

Monday Morning, November 3, 2014

$\begin{array}{ll}\text { 7:30-9:00 Ам } & \text { Breakfast - Crocker Dining Hall } \\ \text { 8:00 AM-6:00 PM } & \text { Registration } \\ \text { 8:15-9:45 Aм } & \text { MAla - Conference Welcome and Plenary Session — Chapel } \\ \text { 9:45-10:15 AM } & \text { Coffee Social }\end{array}$

10:15-11:55 AM MORNING SESSIONS

MA1b Learning and Optimization for Big Data

MA2b EEG Based Brain Computer Interface

MA3b Underwater Wireless Networks

MA4b Physical Layer Security I

MA5b Image and Video Processing

MA6b Sparse Estimation and Learning in Multi-Channel and Array Systems

MA7b Architectures for Detection and Decoding

MA8b1 Synchronization and Channel Estimation (Poster)

MA8b2 Relaying (Poster)

MA8b3 Active Sensing and Target Recognition (Poster)

MA8b4 Physiological Signal Processing (Poster)

12:00-1:00 PM $\quad$ Lunch - Crocker Dining Hall

Monday Afternoon, November 3, 2014
1:30-5:10 PM
AFTERNOON SESSIONS
MP1a Big Data Analytics
MP1b Tensor-Based Signal Processing
MP2a Neural Engineering and Signal Processing
MP2b Brain Connectomics
MP3a Compressed Sensing I
MP3b Compressed Sensing II
MP4a Underwater Acoustic Communications and Networking
MP4b Massive MIMO I
MP5a Smart Grid: Learning and Optimization
MP5b Image and Video Quality
MP6a Array Calibration
MP6b Wireless Localization
MP7a Resource-aware and Domain-specific Computing
MP7b Detection and Estimation for Networked Data
MP8a1 Network Resource Allocation and Localization (Poster)
MP8a2 Bioinformatics and Medical Imaging (Poster)
MP8a3 Source Separation and Array Processing (Poster)
MP8a4 Digital Communications (Poster)
MP8a5 Image and Speech Processing (Poster)

Monday Evening, November 3, 2014

6:00-9:30 PM Conference Cocktail/Social — Merrill Hall The Cocktail/Social takes the place of Monday's dinner. No charge for conference attendees and a guest. 


\section{Asilomar Conference Session Schedule (continued)}

Tuesday Morning, November 4, 2014

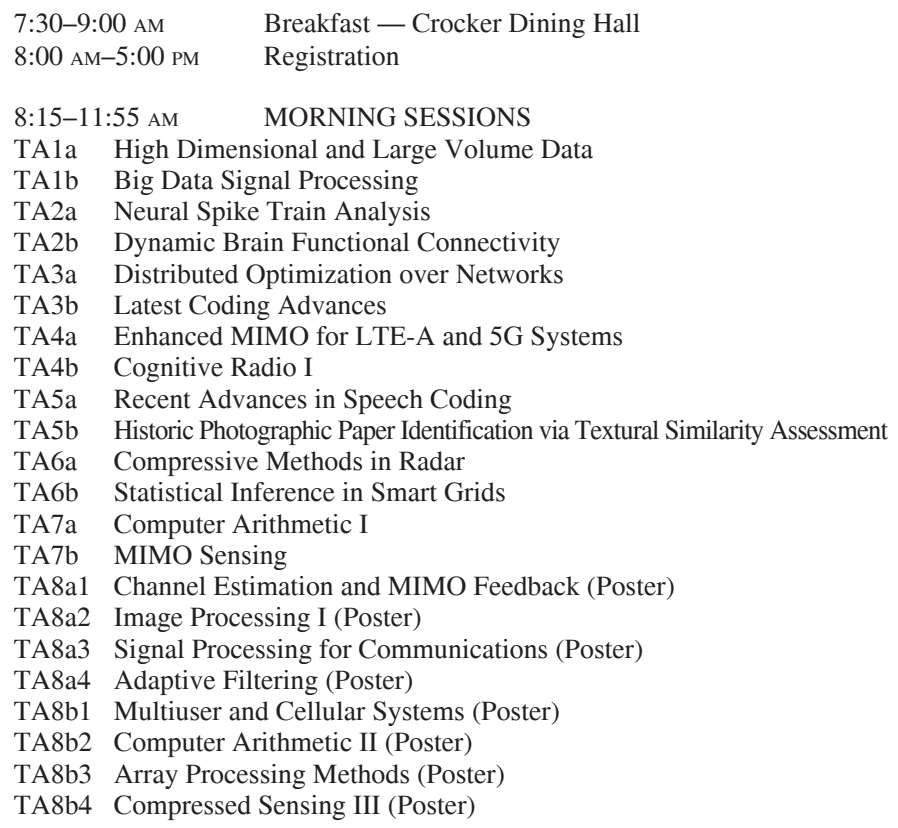

Tuesday Afternoon, November 4, 2014

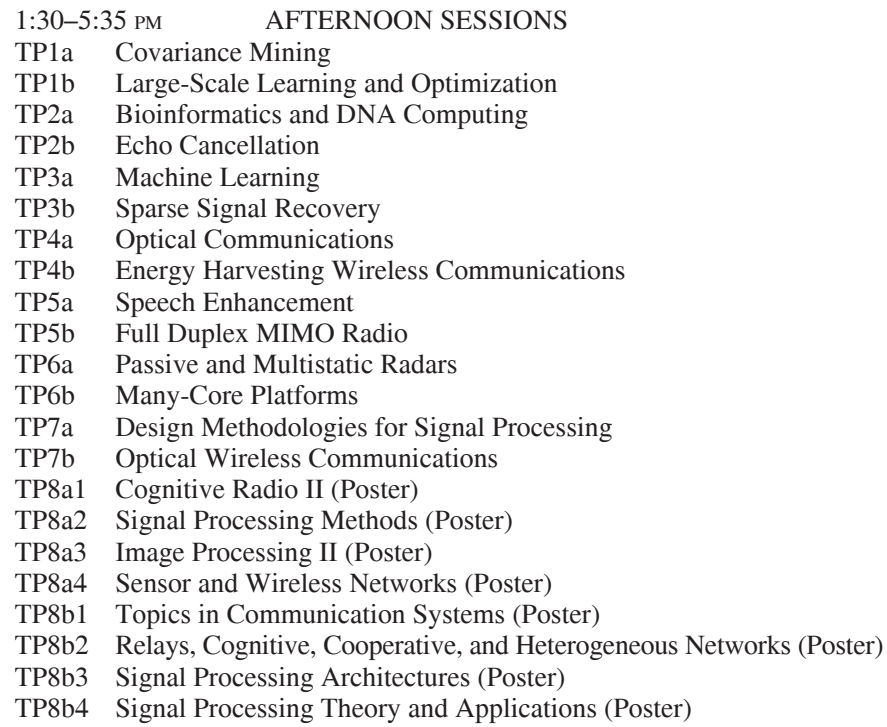




\section{Asilomar Conference Session Schedule (continued)}

Wednesday Morning, November 5, 2014

$\begin{array}{ll}\text { 7:30-9:00 AM } & \text { Breakfast - Crocker Dining Hall } \\ \text { 8:00 AM-12:00 PM } & \text { Registration - Copyright forms must be turned in } \\ & \text { before the registration closes at 12:00 noon. }\end{array}$

8:15-11:55 AM MORNING SESSIONS

WA1a MIMO Design for mmWave Systems

WA1b Massive MIMO II

WA2a 5G and Energy Efficient Cellular Networks

WA2b Mobile Health

WA3a Sparse Learning and Estimation

WA3b Advances in Statistical Learning

WA4a Physical Layer Security II

WA4b Coding and Decoding

WA5a Information Processing for Social and Sensor Networks

WA5b Document Processing and Synchronization

WA6a Adaptive Signal Design and Analysis

WA6b Distributed Detection and Optimization

WA7a Implementation of Wireless Systems

WA7b Video Coding Architecture and Design

12:00-1:00 PM Lunch - Meal tickets may be purchased at registration desk. This meal is not included in the registration. 


\section{Student Paper Contest}

Heather - Sunday, November 2, 2014, 4:00-6:30 PM

Track A

"Everlasting Secrecy in Disadvantaged Wireless Environments against Sophisticated Eavesdroppers"

Azadeh Sheikholeslami, Dennis Goeckel, Hossein Pishro-nik, UMASS-

Amherst, United States

"On Physical Layer Secrecy of Collaborative Compressive Detection"

Bhavya Kailkhura, Thakshila Wimalajeewa, Pramod Varshney, Syracuse University, United States

Track B

"Max-Min Fairness in Compact MU-MIMO Systems: Can the Matching Network Play a Role?"

Yahia Hassan, Armin Wittneben, ETH Zurich, Switzerland

Track C

"On the Convergence Rate of Swap-collide Algorithm for Simple Task Assignment"

Sam Safavi, Usman A. Khan, Tufts University, United States

"Secrecy Outage Analysis of Cognitive Wireless Sensor Networks"

Satyanarayana Vuppala, Jacobs University Bremen, Germany; Weigang Liu, Tharmalingam Ratnarajah, University of Edinburgh, United Kingdom; Giuseppe Abreu, Jacobs University Bremen, Germany

Track D

"Subspace Learning from Extremely Compressed Measurements"

Martin Azizyan, Akshay Krishnamurthy, Aarti Singh, Carnegie Mellon

University, United States

"Abstract Algebraic-Geometric Subspace Clustering”

Manolis Tsakiris, Rene Vidal, Johns Hopkins University, United States

Track E

"Calibrating Nested Sensor Arrays with Model Errors"

Keyong Han, Peng Yang, Arye Nehorai, Washington University in St. Louis, United States

Track F

"Whitening 1/f-type Noise in Electroencephalogram Signals for Steady-State Visual Evoked Potential Brain-Computer Interfaces"

Alan Paris, Azadeh Vosoughi, George Atia, University of Central Florida, United States

Track G

"Hybrid Floating-Point Modules with Low Area Overhead on a Fine-Grained Processing Core"

Jon Pimentel, Bevan Baas, University of California, Davis, United States

Track H

"Crowdsourced Study of Subjective Image Quality"

Deepti Ghadiyaram, Alan Bovik, University of Texas at Austin, United States 


\title{
2014 Asilomar Conference Session Schedule
}

Coffee breaks will be at 9:55 AM and 3:10 PM. (except Monday morning when refreshments will be served outside Merrill Hall from 9:45-10:15 AM)

Monday, November 3, 2014

CONFERENCE WELCOME AND PLENARY SESSION 8:15-9:45 AM

1. Welcome from the General Chairperson

Prof. Roger Woods

Queen's University of Belfast

2. Session MA1a Distinguished Lecture for the 2014 Asilomar Conference

\section{Learning Tools for Big Data Analytics}

Georgios B. Giannakis

Univ. of Minnesota, USA

\begin{abstract}
We live in an era of data deluge. Pervasive sensors collect massive amounts of information on every bit of our lives, churning out enormous streams of raw data in various formats. Mining information from unprecedented volumes of data promises to limit the spread of epidemics and diseases, identify trends in financial markets, learn the dynamics of emergent socialcomputational systems, and also protect critical infrastructure including the smart grid and the Internet's backbone network. While Big Data can be definitely perceived as a big blessing, big challenges also arise with large-scale datasets. The sheer volume of data makes it often impossible to run analytics using a central processor and storage, and distributed processing with parallelized multi-processors is preferred while the data themselves are stored in the cloud. As many sources continuously generate data in real time, analytics must often be performed "on-the-fly" and without an opportunity to revisit past entries. Due to their disparate origins, massive datasets are noisy, incomplete, prone to outliers, and vulnerable to cyber-attacks. These effects are amplified if the acquisition and
\end{abstract}


transportation cost per datum is driven to a minimum. Overall, Big Data present challenges in which resources such as time, space, and energy, are intertwined in complex ways with data resources. Given these challenges, ample signal processing opportunities arise. This keynote lecture outlines ongoing research in novel models applicable to a wide range of Big Data analytics problems, as well as algorithms to handle the practical challenges, while revealing fundamental limits and insights on the mathematical trade-offs involved.

\section{Biography}

Georgios B. Giannakis received his Diploma in Electrical Engineering from the National Technical University of Athens, Greece, 1981. From 1982 to 1986 he was with the University of Southern California, where he received his MSc. in Electrical Engineering (1983), MSc. in Mathematics (1986), and Ph.D. in Electrical Engineering (1986). He became a Fellow of the IEEE in 1997. Since 1999, he has been a Professor with the University of Minnesota where he now holds an ADC Chair in Wireless Telecommunications in the ECE Department, and serves as director of the Digital Technology Center. His general interests span the areas of communications, networking and statistical signal processing - subjects on which he has published more than 370 journal papers, 630 conference papers, 20 book chapters, two edited books and two research monographs (h-index 108). Current research focuses on sparsity and big data analytics, wireless cognitive radios, mobile ad hoc networks, renewable energy, power grid, gene-regulatory, and social networks. He is the (co-) inventor of 22 patents issued, and the (co-) recipient of 8 best paper awards from the IEEE Signal Processing (SP) and Communications Societies, including the G. Marconi Prize Paper Award in Wireless Communications. He also received Technical Achievement Awards from the SP Society (2000), from EURASIP (2005), a Young Faculty Teaching Award, and the G. W. Taylor Award for Distinguished Research from the University of Minnesota. He is a Fellow of EURASIP, and has served the IEEE in a number of posts, including that of a Distinguished Lecturer for the IEEE-SP Society. 



\title{
Program of the 2014 Asilomar Conference on Signals, Systems, and Computers
}

\author{
Technical Program Chairman \\ Prof. Geert Leus \\ Delft University of Technology
}




\section{Session MA1b Learning and Optimization for Big Data}

Co-Chairs: Konstantinos Slavakis, University of Minnesota and Nicholas D. Sidiropoulos, University of Minnesota

MA1b-1 FLEXA: A Fast Parallel Algorithm for $\quad$ 10:15 AM Big-Data Optimization

Francisco Facchinei, Simone Sagratella, University of Rome, Italy; Gesualdo Scutari, University of Buffalo, the State University of New York, United States

MA1b-2 Fast and Robust Bootstrap in Analysing Large 10:40 AM Multivariate Datasets

Shahab Basiri, Esa Ollila, Visa Koivunen, Aalto University, Finland

MA1b-3 Clustering High-Dimensional Dynamical Systems on Low-Rank Matrix Manifolds

Konstantinos Slavakis, X. Wang, G. Lerman, University of Minnesota, United States

MA1b-4 Adaptive Estimation from Big Data via 11:30 AM Censored Stochastic Approximation Dimitrios Berberidis, University of Minnesota, Twin Cities, United States; Gang Wang, Beijing Institute of Technology, China; Georgios Giannakis, Vassilis Kekatos, University of Minnesota, Twin Cities, United States

\section{Session MA2b EEG Based Brain Computer Interface}

\section{Chair: Murat Akcakaya, Northeastern University}

MA2b-1 Decoding the Focus of Auditory Attention 10:15 AM from Single-Trial EEG Signals

Lenny Varghese, Inyong Choi, Siddharth Rajaram, Courtney Pacheco, Barbara Shinn-Cunningham, Boston University, United States

MA2b-2 Auditory Considerations for a Motor Imagery 10:40 AM Brain-Computer Interface for Speech Synthesizer Control Jonathan Brumberg, Jeremy Burnison, University of Kansas, United States

MA2b-3 Single-Trial Identification of Failed Memory 11:05 AM Retrieval

Eunho Noh, University of California, San Diego, United States; Matthew Mollison, Tim Curran, University of Colorado Boulder, United States; Virginia de Sa, University of California, San Diego, United States

MA2b-4 Utilization of Temporal Trial Dependency in 11:30 AM ERP based BCIs

Umut Orhan, CorTech, LLC, United States; Delia Fernandez-Canellas, Universitat Politècnica de Catalunya, Spain; Murat Akcakaya, Dana H. Brooks, Deniz Erdogmus, Northeastern University, United States 


\section{Session MA3b Underwater Wireless Networks}

Chair: Milica Stojanovic, Northeastern University

MA3b-1 On the Feasibility of Fully Wireless Remote 10:15 AM Control for ROVs

Federico Favaro, Filippo Campagnaro, Paolo Casari, Michele Zorzi, University of Padova, Italy

MA3b-2 Modeling Realistic Underwater Acoustic 10:40 AM Networks using Experimental Data Mandar Chitre, Gabriel Chua, National University of Singapore, Singapore

MA3b-3 Scalable Collision-Tolerant Localization in 11:05 AM Underwater Acoustic Sensor Networks Hamid Ramezani, Geert Leus, Technical University of Delft, Netherlands; Milica Stojanovic, Northeastern University, United States

MA3b-4 New Frontiers in Underwater Acoustic 11:30 AM Communications Andrew Singer, Thomas Riedl, University of Illinois at Urbana Champaign, United States

\section{Session MA4b Physical Layer Security I}

Chair: Pramod Varshney, Syracuse University

MA4b-1 On Physical Layer Secrecy of Collaborative 10:15 AM Compressive Detection Bhavya Kailkhura, Thakshila Wimalajeewa, Pramod Varshney, Syracuse University, United States

MA4b-2 Converse Results for Secrecy Generation over 10:40 AM Channels

Himanshu Tyagi, University of California, San Diego, United States; Shun Watanabe, University of Tokushima, Japan

MA4b-3 Robust Transmission over Wiretap Channels 11:05 AM with Secret Keys

Rafael F. Schaefer, H. Vincent Poor, Princeton University, United States

MA4b-4 Secret Key-Private Key Generation for $\quad$ 11:30 AM Multiple Terminals

Huishuai Zhang, Syracuse University, United States;

Lifeng Lai, Worcester Polytechnic Institute, United States; Yingbin Liang, Huishuai Zhang, Syracuse University, United States

\section{Session MA5b Image and Video Processing}

Chair: Marios S. Pattichis, University of New Mexico

MA5b-1 Robust Image Recognition by Multi-Kernel 10:15 AM Dictionary Learning Rituparna Sarkar, Sedat Ozer, Scott Acton, Kevin Skadron, University of Virginia, United States 
Target Tracking

Chuong Nguyen, Joseph Havlicek, University of

Oklahoma, United States; Guoliang Fan, Oklahoma State

University, United States; John Caulfield, Cyan Systems,

United States; Marios Pattichis, University of New

Mexico, United States

MA5b-3 Crowdsourced Study of Subjective Image 11:05 AM

Quality

Deepti Ghadiyaram, Alan Bovik, University of Texas at

Austin, United States

MA5b-4 Detecting Coronal Holes for Solar Activity 11:30 AM

Modeling

Marios Pattichis, University of New Mexico, United

States; Rachel Hock, AFRL/RVBXS Space Vehicles

Directorate, United States; Venkatesh Jatla, University of

New Mexico, United States; Carl Henney, Charles Arge,

AFRL/RVBXS Space Vehicles Directorate, United States

\section{Session MA6b Sparse Estimation and Learning in Multi-Channel and Array Systems}

Co-Chairs: Palghat P. Vaidyanathan, California Institute of Technology and Piya Pal, University of Maryland

MA6b-1 Characterization of Orthogonal Subspaces for 10:15 AM Alias-Free Reconstruction of Damped Complex Exponential Modes in Sparse Arrays

Pooria Pakrooh, Ali Pezeshki, Louis L. Scharf, Colorado State University, United States

MA6b-2 Exploiting Sparsity during the detection of 10:40 AM High-Order QAM Signals in Large Dimension MIMO Systems

Oleg Tanchuk, Bhaskar Rao, University of California, San Diego, United States

MA6b-3 Structured Sparse Representation with 11:05 AM Low-Rank Interference

Minh Dao, Yuanming Suo, Sang (Peter) Chin, Trac Tran, Johns Hopkins University, United States

MA6b-4 Grid-Less Algorithms for Identifying More 11:30 AM Spectral Lines Than Sensors.

Piya Pal, University of Maryland, College Park, United States; P. P. Vaidyanathan, California Institute of Technology, United States

\section{Session MA7b Architectures for Detection and Decoding}

Chair: Joseph R. Cavallaro, Rice University

MA7b-1 A Reduced-Complexity Iterative Decoding 10:15 AM Scheme for Quasi-Cyclic Low-Density ParityCheck Codes

Shu Lin, Keke Liu, Juane Li, University of California, Davis, United States 
MA7b-2 Efficient Adaptive List Successive

Cancellation Decoder for Polar Codes

Chuan Zhang, National Mobile Communications

Research Laboratory, China; Zhongfeng Wang, Broadcom

Corporation, United States; Xiaohu You, National Mobile

Communications Research Laboratory, China

MA7b-3 Decoder Diversity Architectures for Finite 11:05 AM

Alphabet Iterative Decoders for LDPC Codes

Bane Vasic, University of Arizona, United States; David

Declercq, Universite de Cergy-Pontoise, France; Shiva

Planjery, Codelucida, United States

MA7b-4 Asynchronous Design for Precision-Scaleable 11:30 AM

Energy-Efficient LDPC Decoder

Jingwei Xu, Tiben Che, Ehsan Rohani, Gwan Choi, Texas

A\&M university, United States

\section{Session MA8b1 Synchronization and Channel Estimation}

Chair: Shengli Zhou, University of Connecticut

10:15 AM-11:55 AM

MA8b1-1 Frequency Tracking with Intermittent Wrapped Phase Measurement Using the Rao-Blackwellized Particle Filter

Maryam Eslami Rasekh, Upamanyu Madhow, University of California, Santa Barbara, United States; Raghuraman Mudumbai, University of Iowa, United States

MA8b1-2 Improving IEEE 1588v2 Time Synchronization Performance with Phase Locked Loop

Rico Jahja, Suk-seung Hwang, Goo-Rak Kwon, Jae-young Pyun, Seokjoo Shin, Chosun University, Indonesia

MA8b1-3 Superimposed Pilots based Secure Communications for Multiple Antenna System

Yejian Chen, Bell Laboratories, Alcatel-Lucent, Germany

MA8b1-4 An Improved ESPRIT-Based Blind CFO Estimation Algorithm In OFDM Systems

Yen-Chang Pan, See-May Phoong, National Taiwan

University, Taiwan; Yuan-Pei Lin, National Chiao Tung

University, Taiwan

MA8b1-5 Blind, Low Complexity Estimation of Time and Frequency Offsets in OFDM Systems

Rohan Ramlall, University of California, Irvine, United States

MA8b1-6 Efficient NLOS Optical Wireless Channel Estimation based on Sparse Pulse

Xiaoke Zhang, Chen Gong, Zhengyuan Xu, University of Science and Technology of China, China

MA8b1-7 Channel Estimation and Precoder Design for MillimeterWave Communications: The Sparse Way Philip Schniter, Ohio State University, United States;

Akbar Sayeed, Wisconsin, United States 


\section{Session MA8b2 Relaying}

Chair: Guiseppe Caire, TU Berlin

10:15 AM-11:55 AM

MA8b2-1 Performance Analysis of Fixed Gain MIMO AF Relaying with Co-Channel Interferences

Min Lin, Min Li, PLA University of Science and Technology, China; Wei-Ping Zhu, Concordia University, Canada; Kang An, PLA University of Science and Technology, China

MA8b2-2 On Carrier-Cooperation in Parallel Gaussian MIMO Relay Channels with Partial Decode-and-Forward Christoph Hellings, Wolfgang Utschick, Technische Universität München, Germany

MA8b2-3 Enhanced Relay Cooperation via Rate Splitting Ivana Maric, Dennis Hui, Ericsson, United States

MA8b2-4 Alternate versus Simultaneous Relaying in MIMO Cellular Relay Networks: A Degrees of Freedom Study Aya Salah, Amr El-Keyi, Nile University, Egypt; Mohammed Nafie, Cairo University, Egypt

MA8b2-5 Low-Complexity Two-Way AF MIMO Relay Strategy for Wireless Relay Networks

Kanghee Lee, Republic of Korea Air Force, Republic of Korea; Visvakumar Aravinthan, Sunghoon Moon, Wichita State University, United States; Jongbum Ryou, Sungo Kim, Changki Moon, Inha Hyun, Republic of Korea Air Force, Republic of Korea

MA8b2-6 Blind Self-Interference Cancellation for Full-Duplex Relays

Gustavo Gonzalez, Fernando Gregorio, Juan Cousseau, CONICET - Universidad Nacional del Sur, Argentina

\section{Session MA8b3 Active Sensing and Target Recognition}

Chair: Mark R. Bell, Purdue University

10:15 AM-11:55 AM

MA8b3-1 Proximal Constrained Waveform Design Algorithms for Cognitive Radar STAP

Pawan Setlur, Wright State Research Institute, United States; Muralidhar Rangaswamy, Air Force Research Laboratory, United States

MA8b3-2 The Generalized Sinusoidal Frequency Modulated Waveform for High Duty Cycle Active Sonar David Hague, John Buck, University of Massachusetts Dartmouth, United States

MA8b3-3 Concurrent Exploration of Orthogonal Waveform and Co-Prime Array for Quick and High Resolution Scanning Shuo Yang, Xin Wang, Xuehong Lin, Stony Brook University, United States 
MA8b3-4 On Bayesian Transmit Signal Design using Information Theory

Mir H. Mahmood, NextNav LLC, United States; Mark R.

Bell, Purdue University, United States

MA8b3-5 Improved Distributed Automatic Target Recognition

Performance by Exploiting Dominant Scatterer Spatial

Diversity

John Wilcher, William Melvin, Georgia Tech Research

Institute, United States; Aaron Lanterman, Georgia

Institute of Technology, United States

MA8b3-6 Semi-Supervised Classification of Terrain Features in Polarimetric SAR Images using H/A/alpha and the General Four-Component Scattering Power Decompositions

Stephen Dauphin, Sandia National Laboratories, United States; Margaret Cheney, Colorado State University, United States; Derek West, Robert Riley, Sandia National Laboratories, United States

MA8b3-7 A Super-Resolving Near-Field Holographic Method for Underwater EM Signature Modeling

Hatim Alqadah, Naval Research Laboratory, United

States; Nicolas Valdivia, US Naval Research Laboratory,

United States

MA8b3-8 Limitations and Capabilities of the Fractional

Spectrogram Analysis Tool for SAR-Based Detection of Multiple Vibrating Targets

Adebello Jelili, Balu Santhanam, Majeed Hayat,

University of New Mexico, United States

\section{Session MA8b4 Physiological Signal Processing}

Chair: Alessio Medda, Georgia Tech

10:15 AM-11:55 AM

MA8b4-1 Sample-Based Cross-Frequency Coupling Analysis with CFAR Detection

Charles Creusere, Nathan McRae, Mark Norman, Philip

Davis, New Mexico State University, United States

MA8b4-2 Classification of Human Viewers using SVM

Philip Davis, Charles Creusere, Jim Kroger, New Mexico

State University, United States

MA8b4-3 Activity Recognition using Statistical Gait Parameters from a Single Accelerometer

Andrew Vaughan, Alessio Medda, Brian Liu, Shean

Phelps, Georgia Tech Research Institute, United States

MA8b4-4 Intra-Patient and Inter-Patient Seizure Prediction from Spatial-Temporal EEG Features

Shuoxin Ma, Daniel Bliss, Arizona State University,

United States

MA8b4-5 Effective Connectivity in fMRI from Mutual Prediction Approach

Marisel Villafañe-Delgado, Selin Aviyente, Michigan State

University, United States 
MA8b4-6 Whitening 1/f-type Noise in Electroencephalogram Signals for Steady-State Visual Evoked Potential BrainComputer Interfaces Alan Paris, Azadeh Vosoughi, George Atia, University of Central Florida, United States

MA8b4-7 Adaptive Learning of Behavioral Tasks for Patients with Parkinson's Disease Using Signals from Deep Brain Stimulation

Nazanin Zaker, University of Denver, United States; Arindam Dutta, Alexander Maurer, Arizona State University, United States; Jun Zhang, University of Denver, United States; Sara Hanrahan, Adam Hebb, Colorado Neurological Institute, United States; Narayan Kovvali, Antonia Papandreou-Suppappola, Arizona State University, United States

\section{Session MP1a Big Data Analytics}

Chair: Ali Tajer, Rensselaer Polytechnic Institute

MP1a-1 Universal Sequential Outlier Hypothesis

Testing

Yun Li, Sirin Nitinawarat, Venugopal Veeravalli,

University of Illinois at Urbana-Champaign, United States

MP1a-2 Parsimonious Models for Random Variables 1:55 PM and Stochastic Processes

Weiyu Xu, University of Iowa, United States

MP1a-3 Fundamental Limits on Information-Friction 2:20 PM Energy of Big-Data Computing Majid Mahzoon, Pulkit Grover, Carnegie Mellon University, India

MP1a-4 Quickest Search Over Correlated Sequences Ali Tajer, Wayne State University, United States

\section{Session MP1b Tensor-Based Signal Processing}

Chair: Eric Moreau, University of Toulon

MP1b-1 Memory-Efficient Parallel Computation of 3:30 PM

Tensor and Matrix Products for Big Tensor

Decomposition

Niranjay Ravindran, Nicholas Sidiropoulos, Shaden Smith,

George Karypis, University of Minnesota, United States

MP1b-2 Recent Advances on Tensor Models and their 3:55 PM Relevance for Multidimensional Data Processing

Salah Bourennane, Julien Marot, Ecole Centrale Marseille - Institut Fresnel, France

MP1b-3 Tensor-Based Channel Estimation for 4:20 PM

Non-Regenerative Two-Way Relaying Networks with Multiple Relays Jianshu Zhang, Kristina Naskovska, Martin Haardt, Ilmenau University of Technology, Germany

MP1b-4 Fast Non-Unitary Simultaneous

Diagonalization of Third-Order Tensors

Victor Maurandi, Eric Moreau, University of Toulon, France 


\section{Session MP2a Neural Engineering and Signal Processing}

Chair: Ervin Sejdic, University of Pittsburgh

MP2a-1 Electroencephalography-based Alzheimer's 1:30 PM Disease Diagnosis: Where we are at Now and Where we are Heading Tiago Falk, Institut National de la Recherche Scientifique, Canada

MP2a-2 EEG Event Detection Using Big Data Iyad Obeid, Amir Harati, Joseph Picone, Temple University, United States

MP2a-3 A Source Localization Approach to Creating a 2:20 PM Neural Interface with the Peripheral Nervous

System

Jose Zariffa, Toronto Rehabilitation Institute - University

Health Network, Canada

MP2a-4 A Picture is Worth a Thousand Words: Some 2:45 PM Examples of the Utility of Biomedical Image

Processing in Brain Research Negar Memarian, University of California, Los Angeles, United States

\section{Session MP2b Brain Connectomics}

Chair: Dimitri Van De Ville, EPFL

MP2b-1 Brain-Network Continua Revealed with 3:30 PM Multivariate Performance Metrics.

Stephen Strother, Baycrest and University of Toronto, Canada

MP2b-2 Learning with Multi-Site fMRI Graph Data 3:55 PM Gabriel Castrillon, Seyed-Ahmad Ahmadi, Nassir Navab, Technische Universität München, Germany; Jonas Richiardi, Stanford University, United States

MP2b-3 Using Computer Vision to Understand 4:20 PM Biological Vision

Dmitri Chklovskii, Simons Center for Data Analysis, United States

MP2b-4 Dynamic Functional Connectivity: Probing $\quad$ 4:45 PM Spontaneous Network Reorganization Dimitri Van De Ville, Nora Leonardi, École Polytechnique Fédérale de Lausanne / University of Geneva, Switzerland

\section{Session MP3a Compressed Sensing I}

Chair: Aleksandar Dogandzic, Iowa State University

MP3a-1 Robust Line Spectral Estimation 1:30 PM

Gongguo Tang, Colorado School of Mines, United States;

Parikshit Shah, Badri Bhaskar, University of WisconsinMadison, United States; Benjamin Recht, University of California, Berkeley, United States

MP3a-2 On the Applicability of Matrix Completion on 1:55 PM MIMO Radars

Shunqiao Sun, Athina Petropulu, Rutgers University, United States 
MP3a-3 Subspace Learning from Extremely

Compressed Measurements

Martin Azizyan, Akshay Krishnamurthy, Aarti Singh,

Carnegie Mellon University, United States

MP3a-4 Analysis of Misfocus Effects in Compressive 2:45 PM Optical Imaging

Wenbing Dang, Ali Pezeshki, Randy Bartels, Colorado

State University, United States

\section{Session MP3b Compressed Sensing II}

Chair: George Atia, University of Central Florida

MP3b-1 Filter Design for a Compressive Sensing 3:30 PM

Delay Estimation Framework

Misagh Khayambashi, Lee Swindlehurst, University of

California, Irvine, United States

MP3b-2 Adaptive Sequential Compressive Detection 3:55 PM

Davood Mardani, George Atia, University of Central

Florida, United States

MP3b-3 A Recursive Way for Sparse Reconstruction 4:20 PM of Parametric Spaces

Oguzhan Teke, Bilkent University, Turkey; Ali Cafer

Gurbuz, TOBB University of Economics and Technology,

Turkey; Orhan Arikan, Bilkent University, Turkey

MP3b-4 Subspace Methods for Recovery of Low Rank 4:45 PM \& Joint Sparse Matrices

Sampurna Biswas, Mathews Jacob, Soura Dasgupta,

University of Iowa, United States

\section{Session MP4a Underwater Acoustic Communications and Networking}

Chair: Zhaohui Wang, Michigan Technological University

MP4a-1 Experimental Study of Secret Key Generation 1:30 PM

in Underwater Acoustic Channels

Yi Huang, University of Connecticut, United States;

Lifeng Lai, Worcester Polytechnic Institute, United States;

Shengli Zhou, Zhijie Shi, University of Connecticut,

United States

MP4a-2 Random Linear Packet Coding for Fading 1:55 PM

Channels: Joint Power and Rate Control

Rameez Ahmed, Milica Stojanovic, Northeastern

University, United States

MP4a-3 Underwater Acoustic Communications in

2:20 PM

Great Lakes and in Oceans: What is the Difference?

Wensheng Sun, Mohsen Jamalabdollahi, Zhaohui Wang,

Seyed Zekavat, Michigan Technological University, United

States

MP4a-4 Information-Guided Pilot Insertion for 2:45 PM

Capacity Improvement in OFDM Underwater

Acoustic Communications

Xilin Cheng, Colorado State University, United States;

Miaowen Wen, Xiang Cheng, Peking University, China;

Liuqing Yang, Colorado State University, United States 


\section{Session MP4b Massive MIMO I}

Chair: Erik Larsson, Linköping University

MP4b-1 Jsdm and Multi-Cell Networks: Handling 3:30 PM Inter-Cell Interference Through Long-Term Antenna Statistics Ansuman Adhikary, University of Southern California, United States; Giuseppe Caire, Technical University Berlin, Germany

MP4b-2 Enabling Massive MIMO Systems in the 3:55 PM FDD Mode thanks to D2D Communications

Haifan Yin, Laura Cottatellucci, David Gesbert, Eurecom, France

MP4b-3 Massive MIMO As a Cyber-Weapon Erik G. Larsson, Linkoping University, Sweden; Marcus Karlsson, Linköping University, Sweden

MP4b-4 Large Antenna Array and Propagation 4:45 PM Environment Interaction Xiang Gao, Meifang Zhu, Fredrik Rusek, Fredrik Tufvesson, Ove Edfors, Lund University, Sweden

\section{Session MP5a Smart Grid: Learning and Optimization}

Chair: Gonzalo Mateos, University of Minnesota

MP5a-1 Dynamic Attacks on Power Systems 1:30 PM

Economic Dispatch

Jinsub Kim, Lang Tong, Robert Thomas, Cornell University, United States

MP5a-2 Line Outage Detection in Power Transmission 1:55 PM Networks Via Message Passing Algorithms Jianshu Chen, University of California, Los Angeles, United States; Yue Zhao, Andrea Goldsmith, Stanford University, United States; H. Vincent Poor, Princeton University, United States

MP5a-3 Online Learning Approaches for Dynamic 2:20 PM Optimal Power Flow Seung-Jun Kim, Georgios Giannakis, University of Minnesota, United States

MP5a-4 Decentralized Primary Frequency Control in 2:45 PM Power Networks Changhong Zhao, Steven Low, California Institute of Technology, United States

\section{Session MP5b Image and Video Quality}

Chair: Pamela C. Cosman, University of California, San Diego

MP5b-1 Real-Time 3D Rotation Smoothing for Video 3:30 PM Stabilization Chao Jia, Zeina Sinno, Brian Evans, University of Texas at Austin, United States 
MP5b-2 Joint Source-Channel Rate-Distortion

Optimization with Motion Information Sharing for

H.264/AVC Video-Plus-Depth Coding

Yueh-Lun Chang, University of California, San Diego,

United States; Yuan Zhang, Communication University of

China, China; Pamela Cosman, University of California,

San Diego, United States

MP5b-3 Image Assisted Upsampling of Depth Map via 4:20 PM

Nonlocal Similarity

Wentian Zhou, Xin Li, Daryl Reynolds, West Virginia

University, United States

MP5b-4 Video De-Interlacing Using Asymmetric $\quad$ 4:45 PM

Nonlocal-Means Filtering

Roozbeh Dehghannasiri, Texas A\&M University, United

States

\section{Session MP6a Array Calibration}

Chair: Visa Koivunen, Aalto University

MP6a-1 Bilinear Compressed Sensing for Array 1:30 PM

Self-Calibration

Benjamin Friedlander, University of California, Santa

Cruz, United States; Thomas Strohmer, University of

California, Davis, United States

MP6a-2 Calibrating Nested Sensor Arrays with Model 1:55 PM Errors

Keyong Han, Peng Yang, Arye Nehorai, Washington University in St. Louis, United States

MP6a-3 A New Method for DOA Estimation in the

2:20 PM

Presence of Unknown Mutual Coupling of an

Antenna Array

Eric Wei-Jhong Ding, Borching Su, National Taiwan

University, Taiwan

MP6a-4 An Angular Sampling Theorem for the Usable 2:45 PM Frequency Range of Antenna Array Calibration Measurements

Chung-Cheng Ho, Scott Douglas, Southern Methodist

University, United States

\section{Session MP6b Wireless Localization}

Chair: Petar M. Djuric, Stony Brook University

MP6b-1 Direct Localization of Emitters Using Widely 3:30 PM Spaced Sensors in Multipath Environments Nil Garcia, New Jersey Institute of Technology, United States; Marco Lops, Universita degli Studi di Cassino, Italy; Martial Coulon, University of Toulouse, France; Alexander Haimovich, New Jersey Institute of Technology,

United States; Jason Dabin, Space and Naval Warfare

Systems Command - Systems Center Pacific, United States

MP6b-2 Millimeter-Wave Personal Radars for 3D 3:55 PM Environment Mapping Anna Guerra, Francesco Guidi, Davide Dardari, University of Bologna, Italy 
MP6b-3 Simultaneous Tracking and RSS Model

Calibration by Robust Filtering

Juan Manuel Castro-Arvizu, Universitat Politècnica de Catalunya, Spain; Jordi Vilà-Valls, Pau Closas, Centre Tecnolòic de Telecomunicacions de Catalunya, Spain; Juan Fernández-Rubio, Universitat Politècnica de Catalunya, Spain

MP6b-4 Proximity Detection with RFID in the Internet 4:45 PM of Things

Miodrag Bolic, Majed Rostamian, University of Ottawa, United States; Petar Djuric, Stony Brook University, United States

\section{Session MP7a Resource-aware and Domain- specific Computing}

Chair: Frank Hannig, Friedrich-Alexander University ErlangenNurnberg

MP7a-1 Partial Expansion of Dataflow Graphs for 1:30 PM

Resource-Aware Scheduling of Multicore Signal

Processing Systems

George Zaki, IGI Technologies, United States; William

Plishker, Shuvra Bhattacharyya, University of Maryland,

College Park, United States; Frank Fruth, Texas

Instruments, United States

MP7a-2 Performance Analysis of Weakly-Consistent 1:55 PM Scenario-Aware Dataflow Graphs

Marc Geilen, TU Eindhoven, Netherlands; Joachim Falk, University of Erlangen-Nuremberg, Germany; Christian

Haubelt, Universität Rostock, Germany; Twan Basten, TU Eindhoven, Netherlands; Bart Theelen, TNO-ESI, Netherlands; Sander Stuijk, TU Eindhoven, Netherlands

MP7a-3 Application-driven Reconfiguration of Shared 2:20 PM Resources for Timing Predictability of MPSoC Platforms

Deepak Gangadharan, Ericles Sousa, Vahid Lari,

Frank Hannig, Juergen Teich, University of ErlangenNuremberg, Germany

MP7a-4 Accelerating the Dynamic Time Warping 2:45 PM Distance Measure using Logarithmic Arithmetic Joseph Tarango, University of California, Riverside / Intel, United States; Eamonn Keogh, Philip Brisk, University of California, Riverside, United States

\section{Session MP7b Detection and Estimation for Networked Data}

Chair: Yue Lu, Harvard University

MP7b-1 Detecting Convoys in Networks of

Short-Range Sensors

Sean Lawlor, Michael Rabbat, McGill University, Canada

MP7b-2 Distributed SPRT for Gaussian Binary

Hypothesis Testing: Performance Analysis and

Fundamental Trade-offs

Anit Sahu, Soummya Kar, Carnegie Mellon University,

United States 
MP7b-3 Denoising of Network Graphs using Topology 4:20 PM Diffusion

Mohammad Aghagolzadeh, Hayder Radha, Michigan

State University, United States

MP7b-4 Optimal Hypothesis Testing with

Combinatorial Structure: Applications in Graph

Detection

Yue M. Lu, Harvard University, United States

\section{Session MP8a1 Network Resource Allocation and Localization}

Chair: Michael Rabbat, McGill University

1:30 PM-3:10 PM

MP8a1-1 Optimal Scheduling Policies and the Performance of the CDF Scheduling

PhuongBang Nguyen, Bhaskar Rao, University of

California, San Diego, United States

MP8a1-2 Joint Interference and User Association Optimization in Cellular Wireless Networks

Changkyu Kim, Russell Ford, Sundeep Rangan, New York

University, Polytechnic School of Engineering, United

States

MP8a1-3 Throughput Maximization in Wireless Powered Communication Networks with Energy Saving Rui Wang, Donald Brown, Worcester Polytechnic Institute, United States

MP8a1-4 Optimal Flow Bifurcation in Networks with Dual Base Station Connectivity and Non-ideal Backhaul Amitav Mukherjee, Hitachi America, Ltd., United States

MP8a1-5 Joint Sequential Target State Estimation and Clock Synchronization in Wireless Sensor Networks Jichuan Li, Arye Nehorai, Washington University in St. Louis, United States

MP8a1-6 High-Accuracy Vehicle Position Estimation Using a Cooperative Algorithm with Anchors and Probe Vehicles Ramez L. Gerges, First Responder System Testbed (FiRST), United States; John J. Shynk, University of California, Santa Barbara, United States

MP8a1-7 Statistical Scheduling of Economic Dispatch and Energy Reserves of Hybrid Power Systems with High Renewable Energy Penetration Yi Gu, Huaiguang Jiang, University of Denver, United States; Yingchen Zhang, National Renewable Energy Laboratory, United States; David Wenzhong Gao, University of Denver, United States 


\section{Session MP8a2 Bioinformatics and Medical Imaging}

Chair: George Atia, University of Central Florida

1:30 PM-3:10 PM

MP8a2-1 Comparison and Integration of Genomic Profiles Predict Brain Cancer Survival and Drug Targets Katherine Aiello, Orly Alter, University of Utah, United States

MP8a2-2 Tensor GSVD for Comparison of Two Large-Scale Multidimensional Datasets

Theodore Schomay, Preethi Sankaranarayanan, Katherine Aiello, Orly Alter, University of Utah, United States

MP8a2-3 An Efficient ADMM-based Sparse Reconstruction Strategy for Multi-Level Sampled MRI

Joshua Trzasko, Eric Borisch, Paul Weavers, Armando Manduca, Phillip Young, Stephen Riederer, Mayo Clinic, United States

MP8a2-4 Multiscale Functional Networks in Human Resting State Functional MRI

Jacob Billings, Emory University, United States; Alessio Medda, Georgia Tech Research Institute, United States; Shella Keilholz, Georgia Institute of Technology / Emory University, United States

MP8a2-5 Piecewise Linear Slope Estimation Atul Ingle, William Sethares, Tomy Varghese, James Bucklew, University of Wisconsin-Madison, United States

MP8a2-6 Fast Magnetic Resonance Parametric Imaging via Model-Based Low-Rank Matrix Factorization Parisa Amiri Eliasi, New York University, Polytechnic School of Engineering, United States; Li Feng, Ricardo Otazo, New York University, School of Medicine, United States; Sundeep Rangan, New York University, Polytechnic School of Engineering, United States

MP8a2-7 A Signal Model for Forensic DNA Mixtures Ullrich Mönich, Massachusetts Institute of Technology, United States; Catherine Grgicak, Boston University, United States; Viveck Cadambe, Yonglin Wu, Massachusetts Institute of Technology, United States; Genevieve Wellner, Boston University, United States; Ken Duffy, National University of Ireland Maynooth, Ireland; Muriel Médard, Massachusetts Institute of Technology,

United States

\section{Session MP8a3 Source Separation and Array Processing}

Chair: Douglas Cochran, Arizona State University

$$
\text { 1:30 PM-3:10 PM }
$$

MP8a3-1 Forward - Backward Greedy Algorithms for Signal Demixing Nikhil Rao, Parikshit Shah, Stephen Wright, University of Wisconsin, United States 
MP8a3-2 An Extended Family of Bounded Component Analysis Algorithms

Huseyin Atahan Inan, Alper Tunga Erdogan, Koc

University, Turkey

MP8a3-3 Source Separation in Noisy and Reverberant Environment using Miniature Microphone Array

Shuo Li, Milutin Stanacevic, Stony Brook University,

United States

MP8a3-4 Competitive Algorithm Blending for Enhanced Source Separation

Keith Gilbert, Karen Payton, University of Massachusetts Dartmouth, United States

MP8a3-5 Design of Coprime DFT Arrays and Filter Banks Chun-Lin Liu, P. P. Vaidyanathan, California Institute of Technology, United States

MP8a3-6 The Differential Geometry of Asymptotically Efficient Subspace Estimation

Thomas Palka, Raytheon, United States; Richard Vaccaro, University of Rhode Island, United States

MP8a3-7 Effects of Network Topology on the Conditional Distributions of Surrogated Generalized Coherence Estimates

Lauren Crider, Douglas Cochran, Arizona State University, United States

MP8a3-8 Maximum Energy Sequential Matrix Diagonalisation for Parahermitian Matrices

Jamie Corr, Keith Thompson, Stephan Weiss, University of Strathclyde, United Kingdom; John McWhirter, Cardiff University, United Kingdom; Ian Proudler, Loughbourgh

University, United Kingdom

\section{Session MP8a4 Digital Communications}

Chair: James Glenn-Anderson, Supercomputer Systems Inc. 1:30 PM-3:10 PM

MP8a4-1 High-throughput DOCSIS Upstream QC-LDPC Decoder Bei Yin, Michael Wu, Rice University, United States; Christopher Dick, Xilinx Incorporated, United States; Joseph R. Cavallaro, Rice University, United States

MP8a4-2 On the Performance of LDPC and Turbo Decoder Architectures with Unreliable Memories Joao Andrade, Instituto de Telecomunicações, Universidade de Coimbra, Portugal; Aida Vosoughi, Guohui Wang, Rice University, United States; Georgios Karakonstantis, Andreas Burg, Telecommunication Circuits Lab, EPFL, Switzerland; Gabriel Falcao, Vitor Silva, Instituto de Telecomunicações, Universidade de Coimbra, Portugal; Joseph R. Cavallaro, Rice University, United States

MP8a4-3 Successive Cancellation List Polar Decoder using Loglikelihood Ratios Bo Yuan, Keshab K. Parhi, University of Minnesota, Twin Cities, United States 
MP8a4-4 60 GHz Synthetic Aperture Radar for Short-Range Imaging: Theory and Experiments

Babak Mamandipoor, University of California, Santa Barbara, United States; Greg Malysa, Amin Arbabian, Stanford University, United States; Upamanyu Madhow, University of California, Santa Barbara, United States; Karam Noujeim, Anritsu Co., United States

MP8a4-5 A Systematic Procedure for Deriving Block-Parallel, Power Efficient, Digital Filter Architectures for HighSpeed Data Conversion

Paraskevas Argyropoulos, Hanoch Lev-Ari, Northeastern University, United States

MP8a4-6 Distributed Synchronization of a Testbed Network with USRP N200 Radio Boards

Gilberto Berardinelli, Jakob L. Buthler, Fernando M.

L. Tavares, Oscar Tonelli, Dereje A. Wassie, Farhood Hakhamaneshi, Troels B. Sørensen, Preben Mogensen, Aalborg University, Denmark

MP8a4-7 Design Study of a Short-Range Airborne UAV Radar for Human Monitoring

Sevgi Zubeyde Gurbuz, TOBB University of Economics and Technology, Turkey; Muhsin Alperen Bolucek, Tunahan Kirilmaz, TUALCOM Communication and RF Technologies, Turkey; Unver Kaynak, TOBB University of Economics and Technology, Turkey

MP8a4-8 Max-Min Fairness in Compact MU-MIMO Systems: Can the Matching Network Play a Role?

Yahia Hassan, Armin Wittneben, ETH Zurich, Switzerland

\section{Session MP8a5 Image and Speech Processing}

Chair: Linda S. DeBrunner, Florida State University

$$
\text { 1:30 PM-3:10 PM }
$$

MP8a5-1 Acoustic Echo and Noise Cancellation using Kalman Filter in a Modified GSC Framework Subhash Tanan, Karan Nathwani, Ayush Jain, Rajesh M Hegde, Indian Institute of Technology Kanpur, India; Ruchi Rani, Abhijit Tripathy, Samsung R\&D Institute India Delhi, India

MP8a5-2 Paper Texture Classification via Multi-Scale Restricted Boltzman Machines Arash Sangari, William Sethares, University of WisconsinMadison, United States

MP8a5-3 Regularized Logistic Regression Based classification for Infrared Images

Golrokh Mirzaei, Mohsin M. Jamali, University of Toledo, United States; Jeremy Ross, Peter Gorsevski, Verner Bingman, Bowling Green State University, United States

MP8a5-4 Localizing Near and Far Field Acoustic Sources with Distributed Microphone Arrays Martin Weiss Hansen, Jesper Rindom Jensen, Mads Grcesbøll Christensen, Aalborg University, Denmark 
MP8a5-5 Graph Wavelet Transform: Application to Image

Segmentation

Alp Ozdemir, Selin Aviyente, Michigan State University,

United States

MP8a5-6 Histogram Transform Model Using MFCC Features for

Text-Independent Speaker Identification

Hong Yu, Zhanyu Ma, Beijing University of Posts and

Telecommunications, China; Minyue Li, Jun Guo, Google,

Inc., Sweden

\section{Session TA1a High Dimensional and Large Volume Data}

Chair: Sergiy Vorobyov, Aalto University

TA1a-1 Tensor Restricted Isomety Property for

Multilinear Sparse System of Genomic Interactions

Alexandra Fry, Carmeliza Navasca, University of

Alabama at Birmingham, United States

TA1a-2 Analysis of a Separable STAP Algorithm for 8:40 AM

Very Large Arrays

Jie Chen, Feng Jiang, A. Lee Swindlehurst, University of

California, Irvine, United States

TA1a-3 Spatial-Temporal Characterization of

9:05 AM

Synchrophasor Measurement Systems - A Big

Data Approach for Smart Grid System Situational

Awareness

Huaiguang Jiang, University of Denver, United States; Lei

Huang, Electric Power Research Institute, China Southern

Power Grid, China; Jun Zhang, University of Denver,

United States; Yingchen Zhang, National Renewable

Energy Laboratory, United States; David Wenzhong Gao,

University of Denver, United States

TA1a-4 Performance Analysis of the Tucker HOSVD 9:30 AM for Extracting Low-Rank Structure from Multiple

Signal-Plus-Noise Matrices

Himanshu Nayar, Rajesh Nadakuditi, University of

Michigan, Ann Arbor, United States

\section{Session TA1b Big Data Signal Processing}

Chair: Georgios B. Giannakis, University of Minnesota

TA1b-1 A Comparison of Clustering and Missing 10:15 AM Data Methods for Health Sciences

Ran Zhao, Claremont Graduate University, United States;

Deanna Needell, Claremont McKenna College, United

States; Christopher Johansen, Jerry Grenard, Claremont

Graduate University, United States

TA1b-2 Discovery of Principles of Nature from 10:40 AM

Matrix and Tensor Modeling of Large-Scale

Molecular Biological Data

Orly Alter, University of Utah, United States

TA1b-3 Big Data Clustering Using Random Sampling 11:05 AM and Consensus

Panagiotis Traganitis, Konstantinos Slavakis, Georgios

Giannakis, University of Minnesota, United States 
Misses

Fatemeh Sheikholeslami, Morteza Mardani, Georgios

Giannakis, University of Minnesota, United States

\section{Session TA2a Neural Spike Train Analysis}

Chair: Rebecca Willett, University of Wisconsin-Madison

TA2a-1 Neural Spike Train Denoising by Point

Process Re-weighted Iterative Smoothing

Demba Ba, Massachusetts Institute of Technology, United

States; Behtash Babadi, University of Maryland, College

Park, United States; Emery Brown, Massachusetts

Institute of Technology / Harvard University, United States

TA2a-2 Neurally Inspired Objective Function for 8:40 AM

Subspace Tracking and Online Feature Learning

Dmitri Chklovskii, Simons Center for Data Analysis,

United States

TA2a-3 Tracking Influence in Dynamic Neural

9:05 AM

Networks

Rebecca Willett, University of Wisconsin-Madison, United

States; Eric Hall, Duke University, United States

TA2a-4 A Design and Implementation Framework for 9:30 AM Unsupervised High-resolution Recursive Filters in

Neuromotor Prosthesis Applications

Islam Badreldin, Karim Oweiss, University of Florida,

United States

\section{Session TA2b Dynamic Brain Functional Connectivity}

Chair: Laleh Najafizadeh, Rutgers University

TA2b-1 Functional Connectivity Differences in Brain 10:15 AM

Networks: Contributions of Shared and Unshared

Variance

Michael Cole, Rutgers University, United States; Grega

Repovs?, University of Ljubljana, United States; Alan

Anticevic, Yale University, United States

TA2b-2 Beyond Brain Maps: Functional Connectivity 10:40 AM versus Task-Based Activations in Mental State

Prediction

Irina Rish, IBM T. J. Watson Research Center, United

States

TA2b-3 Approaches for Capturing Dynamic

11:05 AM

Connectivity States in fMRI data

Vince Calhoun, University of New Mexico, United States

TA2b-4 Characterizing whole Brain Modulatory 11:30 AM Interactions in Resting-State

Bharat Biswal, New Jersey Institute of Technology, United

States 


\section{Session TA3a Distributed Optimization over Networks}

Chair: Philippe Ciblat, TELECOM ParisTech

TA3a-1 The ADMM Algorithm for Distributed

Averaging: Convergence Rates and Optimal

Parameter Selection

Euhanna Ghadimi, Andr'e Teixeira, Royal Institute of

Technology-KTH, Sweden; Michael Rabbat, McGill

University, Canada; Mikael Johansson, Royal Institute of

Technology-KTH, Sweden

TA3a-2 Performance Analysis of Multitask Diffusion 8:40 AM Adaptation Over Asynchronous Networks

Roula Nassif, Cédric Richard, André Ferrari, Université de Nice Sophia-Antipolis, France; Ali H. Sayed, University of California, Los Angeles, France

TA3a-3 On the Convergence of an Alternating 9:05 AM Direction Penalty Method for Nonconvex Problems Sindri Magnússon, P. Chathuranga Weeraddana, KTH Royal Institute of Technology, Sweden; Michael Rabbat, McGill University, Canada; Carlo Fischione, KTH Royal Institute of Technology, Sweden

TA3a-4 Decentralized Regression with Asynchronous 9:30 AM Sub-Nyquist Sampling

Hoi To Wai, Anna Scaglione, University of California, Davis, United States

\section{Session TA3b Latest Coding Advances}

Chair: Hamid Jafarkhani, University of California, Irvine

TA3b-1 Joint Space-Time Code Designs for Multiple 10:15 AM Access Channels

Tianyi Xu, InterDigital Communications, Inc., United

States; Xiang-Gen Xia, University of Delaware, United

States

TA3b-2 Quantized Distributed Reception Techniques 10:40 AM for MIMO Wireless Systems

Junil Choi, David Love, Purdue University, United States

TA3b-3 Generalized Spatial Modulation for 11:05 AM

Large-Scale MIMO Systems: Analysis and

Detection

Theagarajan Lakshmi Narasimhan, Patchava Raviteja,

Ananthanarayanan Chockalingam, Indian Institute of

Science, India

TA3b-4 Bandwidth Analysis of Low-Complexity

11:30 AM

Decoupling Networks for Multiple Coupled

Antennas

Ding Nie, Bertrand Hochwald, University of Notre Dame,

United States 


\section{Session TA4a Enhanced MIMO for LTE-A and 5G Systems}

Chair: Fred Vook, Nokia Siemens Networks

TA4a-1 3D Channel Models for Elevation $8: 15$ AM

Beamforming and FD-MIMO in LTE-A and 5G Jianzhong (Charlie) Zhang, Yang Li, Young-Han Nam, Samsung, United States

TA4a-2 Advanced Antenna Solutions for 5G Wireless 8:40 AM Access Erik Dahlman, Stefan Parkvall, David Astely, Hugo Tullberg, Ericsson, Sweden

TA4a-3 Multi-Layer Precoding for Full-Dimensional 9:05 AM MIMO Systems Ahmed Alkhateeb, University of Texas at Austin, United States; Geert Leus, Delft University of Technology,

Netherlands; Robert W. Heath Jr., University of Texas at Austin, United States

TA4a-4 Massive MIMO for mmWave systems 9:30 AM Frederick Vook, Timothy Thomas, Nokia Solutions and Networks, United States

\section{Session TA4b Cognitive Radio I}

Chair: Paul de Kerret, Eurecom

TA4b-1 Statistically Coordinated Precoding for the 10:15 AM MISO Cognitive Radio Channel Paul de Kerret, Miltiades Filippou, David Gesbert, Eurecom, France

TA4b-2 Simultaneous Detection and Estimation based 10:40 AM Spectrum Sharing in Cognitive Radio Networks Jyoti Mansukhani, Priyadip Ray, Indian Institute of Technology Kharagpur, India; Pramod Varshney, Syracuse University, United States

TA4b-3 Interference-Temperature Limit for Cognitive 11:05 AM Radio Networks with MIMO Primary Users Cristian Lameiro, University of Cantabria, Spain; Wolfgang Utschick, Technische Universität München, Germany; Ignacio Santamaria, University of Cantabria, Spain

TA4b-4 Competitive Dynamic Pricing under Demand 11:30 AM Uncertainty

Yixuan Zhai, Qing Zhao, University of California, Davis, United States

\section{Session TA5a Recent Advances in Speech Coding}

Chair: Tokunbo Ogunfunmi, Santa Clara University

TA5a-1 Large Margin Nearest Neighborhood Metric 8:15 AM Learning for I-Vector Based Speaker Verification Waquar Ahmad, Harish Karnick, Rajesh M Hegde, Indian Institute of Technology Kanpur, India 
TA5a-2

Performance Enhanced Scalable Wideband

Speech Coding for IP Networks

Tokunbo Ogunfunmi, Koji Seto, Santa Clara University,

United States

TA5a-3 Adaptive Control of Applying Band-Width 9:05 AM for Post Filter of Speech Coder Depending on Pitch Frequency

Hironobu Chiba, Univ. of Tsukuba, Japan; Yutaka

Kamamoto, Takehiro Moriya, Noboru Harada, Nippon

Telegraph and Telephone Corp., Japan; Shigeki Miyabe,

Takeshi Yamada, Shoji Makino, Univ. of Tsukuba, Japan

TA5a-4

Classification of Sonorant Consonants

9:30 AM

Utilizing Empirical Mode Decomposition

Ashkan Ashrafi, San Diego State University, United States;

Stanley Wenndt, Air Force Research Laboratory, United

States

\section{Session TA5b Historic Photographic Paper Identification via Textural Similarity Assessment}

Co-Chairs: Andrew G. Klein, Worcester Polytechnic Institute and Patrice Abry, Ecole Superieure de Lyon (CNRS)

TA5b-1 Automated Surface Texture Classification of 10:15 AM Photographic Print Media

Paul Messier, Paul Messier LLC, United States; Richard Johnson, Cornell University, United States

TA5b-2 Eigentextures: An SVD Approach to 10:40 AM Automated Paper Classification William Sethares, Atul Ingle, Tomas Krc, University of Wisconsin, United States; Sally Wood, Santa Clara University, United States

TA5b-3 Texture Classification via Area-Scale 11:05 AM Analysis of Raking Light Images Andrew G. Klein, Western Washington University, United States; Anh Do, Christopher Brown, Worcester Polytechnic Institute, United States; Philip Klausmeyer, WAM, United States

TA5b-4 Hyperbolic Wavelet Transform for Historic 11:30 AM Photographic Paper Classification Challenge Stephane Roux, Patrice Abry, ENS Lyon, France; Herwig Wendt, ENSHEEIT-IRIT, France; Stephane Jaffard, Paris Est University, France

\section{Session TA6a Compressive Methods in Radar}

Chair: Athina Petropulu, Rutgers University

TA6a-1 Sparse Arrays, MIMO, and Compressive 8:15 AM Sensing for GMTI Radar Haley Kim, Alexander Haimovich, New Jersey Institute of Technology, United States 
Calderbank, Duke University, United States

TA6a-3 Robust Multipath Exploitation Radar Imaging 9:05 AM in Urban Sensing Based on Bayesian Compressive Sensing

Qisong Wu, Yimin Zhang, Moeness Amin, Fauzia Ahmad, Villanova University, United States

TA6a-4 Joint Sparse and Low-rank Model for 9:30 AM

Radio-Frequency Interference Suppression in Ultrawideband Radar Applications

Lam Nguyen, Army Research Laboratory, United States; Minh Dao, Trac Tran, Johns Hopkins University, United States

\section{Session TA6b Statistical Inference in Smart Grids}

Co-Chairs: H. Vincent Poor, Princeton University and Yue Zhao, Stanford University

TA6b-1 Revisiting Cyclo-Stationary Random Signal 10:15 AM Analysis for Modeling Renewable Power Masood Parvania, University of California, Davis, United States; Francesco Verde, Universita' Federico II di Napoli, Italy; Anna Scaglione, University of California, Davis, United States; Donatella Darsena, Giacinto Gelli, Universita' Federico II di Napoli, Italy

TA6b-2 Integrating PMU-data-driven and $10: 40 \mathrm{AM}$ Physics-based Analytics for Power Systems Operations

Yang Chen, Le Xie, P. R. Kumar, Texas A\&M University, United States

TA6b-3 Sensor Placement for Real-Time Dynamic 11:05 AM State Estimation in Power Systems: A Structural Systems Approach Pedro Rocha, University of Porto, Portugal; Sergio Pequito, Carnegie Mellon University, United States; Pedro Aguiar, Paula Rocha, University of Porto, Portugal; Soummya Kar, Carnegie Mellon University, United States

TA6b-4 Dynamic Joint Outage Identification and 11:30 AM State Estimation in Power Systems Yue Zhao, Stanford University, United States; Jianshu Chen, University of California, Los Angeles, United States; Andrea Goldsmith, Stanford University, United States; H. Vincent Poor, Princeton University, United States

\section{Session TA7a Computer Arithmetic I}

Chair: Neil Burgess, ARM Inc.

TA7a-1 Ultra-Light Weight Hardware Accelerator 8:15 AM Circuits for Data Encryption in Wearable Systems Sanu Mathew, Sudhir Satpathy, Vikram Suresh, Ram Krishnamurthy, Intel Corporation, United States 
TA7a-3 Low Latency is Low Energy

TA7a-4 Optimizing DSP Circuits by a New Family of 9:30 AM Arithmetic Operators

Javier Hormigo, Julio Villalba, Universidad de Malaga, Spain

\section{Session TA7b MIMO Sensing}

Chair: Jian Li, University of Florida

TA7b-1 Bi-Static MIMO Radar Operations for

Range-Folded Clutter Mitigation

Yuri Abramovich, WR Systems Ltd., United States;

Gordon Frazer, DSTO, Australia; Geoffrey San Antonio,

Naval Research Laboratory, United States; Ben Johnson,

Colorado School of Mines, United States

TA7b-2 Large Phased Array Antenna Calibration 10:40 AM

Using Radar Clutter and MIMO

Matthew Brown, Mitch Mirkin, Dan Rabideau, MIT

Lincoln Laboratory, United States

TA7b-3 High Resolution Imaging for MIMO Forward 11:05 AM Looking Ground Penetrating Radar

Jian Li, Ode Ojowu, Luzhou Xu, University of Florida,

United States; John Anderson, Howard University, United

States; Lam Nguyen, Army Research Laboratory, United

States

TA7b-4 Structure Health Monitoring Exploiting 11:30 AM

Mimo Ultrasonic Sensing and Group Sparse

Bayesian Learning

Qisong Wu, Yimin Zhang, Moeness Amin, Andrew Golato,

Sridhar Santhanam, Fauzia Ahmad, Villanova University,

United States

\section{Session TA8a1 Channel Estimation and MIMO Feedback}

Chair: Ananthanarayanan Chockalingam, Indian Institute of Science

$$
\text { 8:15 AM-9:55 AM }
$$

TA8a1-1 Channel Estimation in Millimeter Wave MIMO Systems with One-Bit Quantization

Jianhua Mo, University of Texas at Austin, United States; Philip Schniter, Ohio State University, United States;

Robert W. Heath Jr., University of Texas at Austin, United States

TA8a1-2 Maximum-Likelihood Joint Channel Estimation and Data Detection for Space Time Block Coded MIMO Systems

Haider Alshamary, Weiyu Xu, University of Iowa, United States 
TA8a1-3 Cramer-Rao Bound for Blind Channel Estimation in Cyclic Prefixed MIMO-OFDM Systems With Few

Received Symbols

Borching Su, Kai-Han Tseng, National Taiwan University,

Taiwan

TA8a1-4 Efficient MIMO Sparse Channel Estimation Using LTE

Sounding Reference Signal

Jeng-Kuang Hwang, Jen-Hao Liu, Chien-Min Chen,

Chuan-Shun Lin, Yuan Ze University, Taiwan

TA8a1-5 Impact of Received Signal on Self-interference Channel Estimation and Achievable Rates in In-band Full-duplex

Transceivers

Dani Korpi, Lauri Anttila, Mikko Valkama, Tampere

University of Technology, Finland

TA8a1-6 MIMO Nullforming with RVQ Limited Feedback and

Channel Estimation Errors

D. Richard Brown III, Worcester Polytechnic Institute,

United States; David Love, Purdue University, United

States

TA8a1-7 Limited Feedback in OFDM Systems for Combating ISI/

ICI Caused by Insufficient Cyclic Prefix Length

Erich Zoechmann, Stefan Pratschner, Stefan Schwarz,

Markus Rupp, Vienna University of Technology, Austria

TA8a1-8 Frugal Channel Tracking for Transmit Beamforming

Omar Mehanna, Nicholas Sidiropoulos, University of

Minnesota, United States

\section{Session TA8a2 Image Processing I}

Chair: Kenneth Jenkins, Pennsylvania State University

\section{8:15 AM-9:55 AM}

TA8a2-1 Second Order Model Deviations of Local Gabor Features for Texture Classification

David Picard, Inbar Fijalkow, ETIS - UMR 8051 / ENSEA,

Université Cergy-Pontoise, CNRS, France

TA8a2-2 Weighted Boundary Matching Error Concealment for HEVC Using Block Partition Decisions

Yan-Tsung Peng, Pamela Cosman, University of

California, San Diego, United States

TA8a2-3 Reducing the Latency and Improving the Resolution of Vector Quantization with Anamorphic Stretch Transform Haochen Yuan, Mohammad H. Asghari, Bahram Jalali, University of California, Los Angeles, United States

TA8a2-4 Supervised Facial Recognition based on Multiresolution Analysis with Radon Transform

Ahmed Aldhahab, George Atia, Wasfy Mikhael, University of Central Florida, United States

TA8a2-5 On Compensating Unknown Pixel Behaviors for Image

Sensors with Embedded Processing

William Guicquero, Michele Benetti, Arnaud Peizerat,

Antoine Dupret, Commissariat à l'énergie atomique et aux énergies alternatives, France; Pierre Vandergheynst, École Polytechnique Fédérale de Lausanne, Switzerland 
TA8a2-6 Representative Selection for Big Data via Sparse Graph and Geodesic Grassmann Manifold Distance

Chinh Dang, Hayder Radha, Michigan State University,

United States

TA8a2-7 A Generic Particle Filtering Approach for Multiple Polyhedral Object Tracking in a Distributed Active Sensor Network

Benoit Fortin, Regis Lherbier, Jea-Charles Noyer, Univ. Littoral Cote d'Opale, France

TA8a2-8 Spatial Domain Synthetic Scene Statistics Debarati Kundu, Brian Evans, University of Texas at Austin, United States

\section{Session TA8a3 Signal Processing for Communications}

Chair: Bhavya Kailkhura, Syracuse University

$$
\text { 8:15 AM-9:55 AM }
$$

TA8a3-1 Energy-Efficient Secure Communications in MISO-SE Systems

Alessio Zappone, Pin-Hsun Lin, Eduard A. Jorswieck, TU Dresden, Germany

TA8a3-2 Distinguishing BFSK from QAM and PSK by Sampling Once per Symbol Mohammad Bari, Milos Doroslovacki, George Washington University, United States

TA8a3-3 Quadratic Program Solution of Communication Links Under Jamming

Koorosh Firouzbakht, Guevara Noubir, Masoud Salehi, Northeastern University, United States

TA8a3-4 An Iterative Soft Decision Based Adaptive K-best Decoder Without SNR Estimation

Mehnaz Rahman, Ehsan Rohani, Gwan Choi, Texas A\&M University, United States

TA8a3-5 MMSE Scaling Enhances Performance in Practical Lattice Codes

Nuwan Ferdinand, University of Oulu, Finland; Matthew Nokleby, Duke University, United States; Brian Kurkoski, Japan Advanced Institute of Science and Technology, Japan; Behnaam Aazhang, Rice University, United States

TA8a3-6 RLS-Based Frequency-domain DFE for Uplink SCFDMA

Naveed Iqbal, Azzedine Zerguine, King Fahd University of Petroleum and Minerals, Saudi Arabia; Naofal Al-Dhahir, University of Texas at Dallas, United States

TA8a3-7 Reduced-State Cyclic Viterbi Receiver for Localized SCFDMA Uplink System

Jeng-Kuang Hwang, Jeng-Da Li, Yu-Chang Hsu, ChuanShun Lin, Yuan-Ze University, Taiwan

TA8a3-8 Energy Detection Using Very Large Antenna Array Receivers Alex Oliveras Martinez, Elisabeth De Carvalho, Petar Popovski, Gert Frølund Pedersen, Aalborg University, Denmark 


\section{Session TA8a4 Adaptive Filtering}

Chair: Milos Doroslovacki, George Washington University

8:15 AM-9:55 AM

TA8a4-1 On Component-Wise Conditionally Unbiased Linear Bayesian Estimation

Mario Huemer, Oliver Lang, Johannes Kepler University

Linz, Austria

TA8a4-2 Performance of Proportionate-type NLMS Algorithm with Gain Allocation Proportional to the Mean Square Weight Deviation

Kevin Wagner, Naval Research Laboratory, United States; Milos Doroslovacki, George Washington University,

United States

TA8a4-4 An Efficient Least Mean Squares Algorithm based on q-Gradient

Ubaid Al-Saggaf, Mohammad Moinuddin, King Abdulaziz University, Saudi Arabia; Azzedine Zerguine, King Fahd University of Petroleum and Minerals, Saudi Arabia

TA8a4-5 Optimal Step Size Control for Acoustic Echo Cancellation

Khosrow Lashkari, Seth Suppappola, Cirrus Logic, United States

TA8a4-6 Stochastic Gradient Algorithm Based on an Improved Higher Order Exponentiated Error Cost Function Umair bin Mansoor, Syed Asad, Azzedine Zerguine, King Fahd University of Petroleum and Minerals, Saudi Arabia

TA8a4-7 Spectral Multiscale Coverage with the Feature Aided CPHD Tracker

Ramona Georgescu, Shuo Zhang, Amit Surana, Alberto Speranzon, Ozgur Erdinc, United Technologies Research Center, United States

TA8a4-8 Adaptive Sampling with Sensor Selection for Target Tracking in Wireless Sensor Networks Abdulkadir Kose, Engin Masazade, Yeditepe University, Turkey

\section{Session TA8b1 Multiuser and Cellular Systems}

Chair: Rafael F. Schaefer, Princeton University

10:15 AM-11:55 AM

TA8b1-1 Average Sum MSE Minimization in the Multi-User Downlink With Multiple Power Constraints Andreas Gründinger, Michael Joham, Technische Universität München, Germany; Jose Pablo Gonzalez Coma, Luis Castedo, University of A Coruna, Spain; Wolfgang Utschick, Technische Universität München, Germany

TA8b1-2 Hierarchical Precoding for Ultra-Dense Heterogeneous Networks Lars Thiele, Martin Kurras, Fraunhofer Institute for Telecommunications Heinrich Hertz Institute, Germany 
TA8b1-3 Detection using Block QR Decomposition for MIMO

HetNets

Robin Thomas, Raymond Knopp, Eurecom, France; Sunil (B.T.) Maharaj, University of Pretoria, South Africa

TA8b1-4 On Performance Prediction for Multiuser Detection

Enabled Systems in Packet Based Asynchronous

Gaussian Multiple Access Channels

Prabahan Basu, MIT Lincoln Laboratory, United States

TA8b1-5 Decentralized Target Rate Optimization for MU-MIMO Leakage Based Precoding

Tim Rüegg, Marc Kuhn, Armin Wittneben, ETH Zurich,

Switzerland

TA8b1-6 Leveraging Interference for Increasing Throughput and Reliability of Commercial Wireless Small Cells Rachel Learned, Michael Pitaro, Matthew Ho,

Massachusetts Institute of Technology, United States

TA8b1-7 Throughput Analysis of LTE and WiFi in Unlicensed Band

Abhijeet Bhorkar, Christian Ibars Casas, Pingping Zong,

Intel Corporation, United States

TA8b1-8 Multi-User Detection for xDSL with Partial Cooperation Among Multiple Operators

Syed Hassan Raza Naqvi, Umberto Spagnolini,

Politecnico di Milano, Italy

\section{Session TA8b2 Computer Arithmetic II}

Chair: Sardar Muhammad Sulaman, Lund University

10:15 AM-11:55 AM

TA8b2-1 Improved Non-restoring Square Root Algorithm with Dual Path Calculation

Kihwan Jun, Earl Swartzlander, University of Texas at Austin, Republic of Korea

TA8b2-2 Merged Residue Number System Generation Michael Sullivan, Earl Swartzlander, University of Texas at Austin, United States

TA8b2-3 Partial Product Generation and Addition for Multiplication in FPGAs With 6-Input LUTs George Walters, Penn State Erie, The Behrend College, United States

TA8b2-4 Low-Power Radix-4 Quotient Generator Milos Ercegovac, University of California, Los Angeles, United States

TA8b2-5 Memristor Based Adders

Divya Mahajan, Matheen Musaddiq, Earl Swartzlander, University of Texas at Austin, United States

TA8b2-6 Canonic Real-Valued FFT Structures

Megha Parhi, Yingjie Lao, Keshab K. Parhi, University of Minnesota, Twin Cities, United States

TA8b2-7 A High Throughput and Low Power Radix-4 FFT Architecture

Soumak Mookherjee, Linda S. DeBrunner, Victor

DeBrunner, Florida State University, United States 
TA8b2-8 A Domain Splitting Algorithm for the Mathematical Functions Code Generator

Olga Kupriianova, Christoph Lauter, UPMC, LIP6,

PEQUAN team, France

\section{Session TA8b3 Array Processing Methods}

Chair: Piya Pal, University of Maryland

10:15 AM-11:55 AM

TA8b3-1 Array Self Calibration with Large Initial Errors

Benjamin Friedlander, University of California, Santa Cruz, United States

TA8b3-2 Maximum Likelihood Estimation for Geolocation in the Presence of Multipath

Benjamin Friedlander, University of California, Santa Cruz, United States

TA8b3-3 Enhanced Location Detection Algorithms Based on Time of Arrival Trilateration

Sajina Pradhan, Jae-young Pyun, Goo-Rak Kwon, Seokjoo

Shin, Suk-seung Hwang, Chosun University, Republic of

Korea

TA8b3-4 Designing Radio Interferometric Positioning Systems for Indoor Localizations in Millimeter Wave Bands Marie Shinotsuka, Georgia Institute of Technology, United States; Yiyin Wang, Shanghai Jiao Tong University, China; Xiaoli Ma, G. Tong Zhou, Georgia Institute of Technology, United States

TA8b3-5 Indoor Sound Source Localization and Number Estimation Using Infinite Gaussian Mixture Model Longji Sun, Qi Cheng, Oklahoma State University, United States

TA8b3-6 On the Structural Nature of Cooperation in Distributed Network Localization

Alireza Ghods, Stefano Severi, Giuseppe Abreu, Jacobs University Bremen, Germany; Samuel Van de Velde, Ghent University, Belgium

TA8b3-7 Enabling Distributed Detection with Dependent Sensors Brian Proulx, Junshan Zhang, Douglas Cochran, Arizona State University, United States

TA8b3-8 Active Sonar Transmission Strategies in the Presence of Strong Direct Blast

Luzhou Xu, Jian Li, Akshay Jain, University of Florida, United States

\section{Session TA8b4 Compressed Sensing III}

Chair: Victor DeBrunner, Florida State University

10:15 AM-11:55 AM

TA8b4-1 Super-resolution Line Spectrum Estimation with Block Priors

Kumar Vijay Mishra, Myung Cho, Anton Kruger, Weiyu $X u$, University of Iowa, United States 
TA8b4-3 Complexity Reduction in Compressive Sensing using Hirschman Uncertainty Structured Random Matrices Peng Xi, Victor DeBrunner, Florida State University, United States

TA8b4-4 A Sparse Approach for Estimation of Amplitude Modulated Sinusoids

Stefan Ingi Adalbjörnsson, Johan Swärd, Andreas Jakobsson, Ted Kronvall, Lund University, Sweden

TA8b4-5 Sparsity Order Estimation for Single Snapshot Compressed Sensing

Florian Roemer, Anastasia Lavrenko, Giovanni Del Galdo, Thomas Hotz, Technische Universitaet Ilmenau, Germany; Orhan Arikan, Bilkent University, Turkey; Reiner Thomae, Technische Universitaet Ilmenau, Germany

TA8b4-6 Streaming Signal Recovery Using Sparse Bayesian Learning

Uditha Wijewardhana, Marian Codreanu, Centre for Wireless Communications, Finland

TA8b4-7 Compressed Change Detection for Structural Health Monitoring

Omid Sarayanibafghi, George Atia, Masoud Malekzadeh, Necati Catbas, University of Central Florida, United States

TA8b4-8 A Sparse Semi-Parametric Chirp Estimator Johan Swärd, Johan Brynolfsson, Andreas Jakobsson, Maria Hansson-Sandsten, Lund University, Sweden

\section{Session TP1a Covariance Mining}

Chair: Pradeep Ravikumar, University of Texas at Austin

TP1a-1 Abstract Algebraic-Geometric Subspace Clustering Manolis Tsakiris, Rene Vidal, Johns Hopkins University, United States

TP1a-2 Minimum Variance Portfolio Optimization 1:55 PM with Robust Shrinkage Covariance Estimation Liusha Yang, Hong Kong University of Science and Technology, Hong Kong SAR of China; Romain Couillet, Supelec, France; Matthew McKay, Hong Kong University of Science and Technology, Hong Kong SAR of China

TP1a-3 Greedy Algorithms in Convex Optimization 2:20 PM on Banach Spaces

Vladimir Temlyakov, University of South Carolina, United States

TP1a-4 Greedy Algorithms for Learning Graphical 2:45 PM Models

Ali Jalali, Christopher Johnson, Pradeep Ravikumar, University of Texas at Austin, United States 


\section{Session TP1b Large-Scale Learning and Optimization}

Chair: Alejandro Ribeiro, University of Pennsylvania

TP1b-1 Distributed Adaptive Sparsity-Imposing

Canonical Correlations

Jia Chen, Ioannis Schizas, University of Texas at

Arlington, United States

TP1b-2 Game-Theoretic Learning In A

3:55 PM

Distributed-Information Setting: Distributed

Convergence To Mean-Centric Equilibria

Brian Swenson, Soummya Kar, Carnegie Mellon

University, United States; Joao Xavier, Instituto Superior

Tecnico, Portugal

TP1b-3 Network Newton

4:20 PM

Aryan Mokhtari, Alejandro Ribeiro, University of

Pennsylvania, United States

TP1b-4 Communication-Computation Tradeoffs in

4:45 PM

Decentralized Stochastic Optimization

Konstantinos Tsianos, Michael Rabbat, McGill University,

Canada

\section{Session TP2a Bioinformatics and DNA} Computing

Co-Chairs: Olgica Milenkovic, University of Illinois at UrbanaChampaign and Farzad Farnoud, California Institute of Technology

TP2a-1 On the Capacity of String-Duplication

1:30 PM

Systems and Genomic Duplication

Farzad Farnoud, California Institute of Technology,

United States; Moshe Schwartz, Ben-Gurion University of the Negev, Israel; Jehoshua Bruck, California Institute of Technology, United States

TP2a-2 Intrinsic Universality and the Computational 1:55 PM Power of Self-Assembly

Damien Woods, California Institute of Technology, United

States

TP2a-3 Hybrid Rank Aggregation for Gene

2:20 PM

Prioritization

Minji Kim, Farzad Farnoud, Olgica Milenkovic,

University of Illinois at Urbana-Champaign, United States

TP2a-4 Rate-Independent Computation in Chemical 2:45 PM

Reaction Networks

David Doty, California Institute of Technology, United

States

\section{Session TP2b Echo Cancellation}

Chair: Steven Grant, Missouri University of Science and Technology

TP2b-1 Echo Cancellation for Bone Conduction 3:30 PM

Transducers

Mohammad Behgam, Steven L. Grant, Missouri University

of Science and Technology, United States 
Control

Gerald Enzner, Rainer Martin, Ruhr-University Bochum, Germany; Peter Vary, RWTH Aachen University, Germany

TP2b-3 A Kalman Filter for Stereophonic Acoustic 4:20 PM Echo Cancellation

Constantin Paleologu, University Politehnica of

Bucharest, Romania; Jacob Benesty, University of Quebec,

Canada; Steven L. Grant, Missouri University of Science and Technology, United States; Silviu Ciochina, University Politehnica of Bucharest, Romania

TP2b-4 Study and Design of Differential Microphone 4:45 PM Array Beamforming

Jingdong Chen, Northwestern Polytechnical University,

China; Jacob Benesty, INRS-EMT, University of Quebec,

Canada

\section{Session TP3a Machine Learning}

Chair: Vassilis Kekatos, University of Minnesota

TP3a-1 Consensus Inference with Multilayer Graphs 1:30 PM for Multi-modal Data

Karthikeyan Natesan Ramamurthy, IBM T. J.

Watson Research Center, United States; Jayaraman

J. Thiagarajan, Lawrence Livermore National

Laboratory, United States; Rahul Sridhar, Premnishanth

Kothandaraman, Ramanathan Nachiappan, SSN College

of Engineering, India

TP3a-2 Energy Price Matrix Factorization

Vassilis Kekatos, University of Minnesota, United States

TP3a-3 A New Reduction Scheme for Gaussian Sum 2:20 PM Filters

Leila Pishdad, Fabrice Labeau, McGill University,

Canada

TP3a-4 Exploring Upper Bounds on the Number of $2: 45$ PM Distinguishable Classes

Catherine Keller, MIT Lincoln Laboratory, United States;

Gary Whipple, Laboratory for Telecommunication

Sciences, United States

\section{Session TP3b Sparse Signal Recovery}

Co-Chairs: Daniel Palomar, Hong Kong University of Science and Technology and Gonzalo Mateos, University of Rochester

TP3b-1 Compression Schemes for Time-Varying 3:30 PM Sparse Signals

Sundeep Prabhakar Chepuri, Geert Leus, Delft University of Technology, Netherlands

TP3b-2 A Fast Algorithm for Sparse Generalized Eigenvalue Problem

Junxiao Song, Prabhu Babu, Daniel Palomar, Hong Kong University of Science and Technology, Hong Kong SAR of China 
Reconstructing Nonnegative Signals with Sparse

Transform Coefficients

Renliang Gu, Aleksandar Dogandžic, Iowa State

University, United States

\section{Session TP4a Optical Communications}

Chair: Philippe Ciblat, TELECOM ParisTech

TP4a-1 Fifth-Order Volterra Series Based Nonlinear 1:30 PM

Equalizer for Long-Haul High Data Rate Optical

Fiber Communications

Abdelkerim Amari, Philippe Ciblat, Yves Jaouen, Telecom

ParisTech, France

TP4a-2 Improving the Ultraviolet Scattering Channel 1:55 PM

Via Beam Reshaping

Difan Zou, Shang-Bin Li, Zhengyuan Xu, School of

Information Science and Technology, and Optical Wireless

Communication and Network Center, China

TP4a-3 Correlation Study on the SIMO Channel

2:20 PM

Output of NLOS Optical Wireless Communications

Boyang Huang, Chen Gong, Zhengyuan Xu, University of

Science and Technology of China, China

TP4a-4 An Improved Performance Decoding

Technique for Asymmetrically and Symmetrically

Clipped Optical (ASCO)-OFDM

Nan Wu, Yeheskel Bar-Ness, New Jersey Institute of

Technology, United States

\section{Session TP4b Energy Harvesting Wireless Communications}

Chair: Sennur Ulukus, University of Maryland

TP4b-1 On the Capacity of the Energy Harvesting 3:30 PM Channel with Energy Transfer

Aylin Yener, Pennsylvania State University, United States

TP4b-2 Sum-rate Analysis for Systems with Wireless 3:55 PM Energy Transfer

Rania Morsi, Derrick Wing Kwan Ng, Robert Schober,

Friedrich-Alexander University of Erlangen-Nuremberg, Germany

TP4b-3 Optimal Energy Routing in Networks with 4:20 PM Energy Cooperation

B. Gurakan, O. Ozel, Sennur Ulukus, University of Maryland, United States

TP4b-4 Renewables Powered Mobile Cloud Offloading

Kaibin Huang, University of Hong Kong, Hong Kong SAR of China 


\section{Session TP5a Speech Enhancement}

Chair: Dalei Wu, Nanjing University of Posts and Telecommunications

TP5a-1 Noise Power Spectral Density Matrix

Estimation Based on Improved IMCRA

Qipeng Gong, Benoit Champagne, Peter Kabal, McGill University, Canada

TP5a-2 BI-CosampSE: Block Identification based 1:55 PM Compressive Sampling Matching Pursuit for Speech Enhancement

Dalei Wu, Nanjing University of Posts and

Telecommunications, China; Wei-Ping Zhu, M.N.S.

Swamy, Concordia university, Canada

TP5a-3 Pitch Estimation for Non-Stationary Speech 2:20 PM Mads Grasbøll Christensen, Jesper Rindom Jensen, Aalborg University, Denmark

TP5a-4 Estimating the Noncircularity of Latent

Components within Complex-Valued Subband

Mixtures with Applications to Speech Processing Greg Okopal, Scott Wisdom, Les Atlas, University of Washington, United States

\section{Session TP5b Full Duplex MIMO Radio}

Chair: Yingbo Hua, University of California, Riverside

TP5b-1 Non-Linear Distortion Cancellation in Full

Digital Domain for Full Duplex Radios

Yang-Seok Choi, Feng Xue, Roya Doostnejad, Shilpa

Talwar, Intel Corporation, United States

TP5b-2 Blind Digital Tuning for Interference

Cancellation in Full-Duplex Radio

Yingbo Hua, University of California, Riverside, United

States

TP5b-3 On In-Band Full-Duplex MIMO Radios with 4:20 PM Transmit and Receive Antenna Reuse

Daniel Bliss, Yu Rong, Arizona State University, United States

TP5b-4 MIMO Broadcast Channel with Continuous $\quad$ 4:45 PM Feedback using Full-duplex Radios

Xu Du, Rice University, United States; Christopher Dick, Xilinx Incorporated, United States; Ashutosh Sabharwal, Rice University, United States

\section{Session TP6a Passive and Multistatic Radars}

Chair: Muralidhar Rangaswamy, Air Force Research Labs

TP6a-1 Passive Multistatic Radar Based on

Long-term Evolution Signals

Sandeep Gogineni, Wright State Research Institute,

United States; Muralidhar Rangaswamy, Wright Patterson Air Force Base - AFRL, United States; Arye Nehorai, Washington University in St. Louis, United States 
TP6a-2 A Correlation-Based Signal Detection

Algorithm in Passive Radar with DVB-T2 Emitter

Guolong Cui, Hongbin Li, Stevens Institute of Technology,

United States; Braham Himed, Air Force Research

Laboratory, United States

TP6a-3 Improving Multistatic MIMO Radar

2:20 PM

Performance in Data-Limited Scenarios

Tariq Qureshi, Muralidhar Rangaswamy, Air Force

Research Laboratory, United States; Kristine Bell, Metron

Inc., United States

TP6a-4 Market based Sensor Mobility Management 2:45 PM for Target Localization

Nianxia Cao, Swastik Brahma, Pramod Varshney,

Syracuse University, United States

\section{Session TP6b Many-Core Platforms}

Chair: Mats Brorsson, KTH

TP6b-1 Towards Modeling and Analyzing

3:30 PM

Performance of LTE Base Station Software

Konstantin Popov, SICS, Sweden; Mats Brorsson, KTH

Royal Institute of Technology, Sweden

TP6b-2 REPLICA T7-16-128 - A 2048-threaded

3:55 PM

16-core 7-FU Chained VLIW Chip Multiprocessor

Martti Forsell, Jussi Roivainen, VTT, Finland

TP6b-3 Improving Image Quality by SSIM Based 4:20 PM Increase of Run-Length Zeros in GPGPU JPEG

Encoding

Stefan Petersson, Håkan Grahn, Blekinge Institute of Technology, Sweden

TP6b-4 Kickstarting High-Performing

4:45 PM

Energy-Efficient Manycore Architectures with

Epiphany

Tomas Nordström, Zain ul-Abdin, Halmstad University,

Sweden; Andreas Olofsson, Adapteva, United States

\section{Session TP7a Design Methodologies for Signal Processing}

Chair: Chris Lee, $N C K U$

TP7a-1 Finding Fast Action Selectors for Dataflow

1:30 PM

Actors

Gustav Cedersjö, Jörn W. Janneck, Jonas Skeppstedt,

Lund University, Sweden

TP7a-2 Automatic Generation of Application Specific 1:55 PM FPGA Multicore Accelerators

Pascal Schleuniger, Andreas Hindborg, Nicklas Bo Jensen, Maxwell Walter, Laust Brock-Nannestad, Lars Bonnichsen, Christian W. Probst, Sven Karlsson, Technical University of Denmark, Denmark

TP7a-3 Dataflow Toolset for Soft-Core Processors on 2:20 PM FPGA for Image Processing Applications

Burak Bardak, Fahad Manzoor Siddiqui, Roger Woods,

Queen's University Belfast, United Kingdom 
Flow

Ryan Marlow, Peter Athanas, Virginia Polytechnic

Institute and State University, United States

\section{Session TP7b Optical Wireless Communications}

Chair: Zhengyuan (Daniel) Xu, University of Science and Technology of China

TP7b-1 Multiuser MISO Indoor Visible Light

Communications

Jie Lian, Mohammad Noshad, Maite Brandt-Pearce,

University of Virginia, United States

TP7b-2 Optical Spatial Modulation OFDM using

Micro LEDs

Muhammad Ijaz, Dobroslav Tsonev, Abdelhamid Younis, University of Edinburgh, United Kingdom; Jonathan J.

D. McKendry, Erdan Gu, Martin Dawson, University of Strathclyde, United Kingdom; Harald Haas, University of Edinburgh, United Kingdom

TP7b-3 Adaptation of OFDM under Visible Light 4:20 PM Communications and Illumination Constraints Thomas Little, Hany Elgala, Boston University, United States

TP7b-4 Hybrid Dimmable Visible Light -with 4:45 PM Infra-Red Optical Wireless Communications Andrew Burton, Z Ghassemlooy, Edward Bently, Hoa LeMinh, Northumbria University, United Kingdom; S K Laiw, National Taiwan University of Science and Technology, Taiwan; Chung Ghiu Lee, Chosun University, Republic of Korea

\section{Session TP8a1 Cognitive Radio II}

Chair: Priyadip Ray, IIT Kharagpur

$$
\text { 1:30 PM-3:10 PM }
$$

TP8a1-1 Characterization of Outage Performance for Cognitive Relay Networks with Mixed Fading Efthymios Stathakis, Lars K. Rasmussen, Mikael Skoglund, Royal Institute of Technology (KTH), Sweden

TP8a1-2 Restless Multi-Armed Bandits under Time-Varying Activation Constraints

Kobi Cohen, Qing Zhao, Anna Scaglione, University of California, Davis, United States

TP8a1-3 On the Optimal Relay Design for Multi-Antenna Cognitive Two-Way AF Relay Networks Maksym Girnyk, KTH Royal Institute of Technology, Sweden; Mikko Vehkaperä, Sergiy Vorobyov, Aalto University, Finland

TP8a1-4 Network Aware Spectrum Efficiency Metric for Heterogeneous and Dynamic Radio Environments Aditya Padaki, Ravi Tandon, Jeffrey Reed, Virginia Polytechnic Institute and State University, United States 
TP8a1-5 A Unified Framework for Robust Cooperative Spectrum Sensing

Qi Cheng, Eric Chan-Tin, Oklahoma State University, United States

TP8a1-6 Receiver Configuration and Testbed Development for Underwater Cognitive Channelization

George Sklivanitis, Emrecan Demirors, Stella N.

Batalama, Tommaso Melodia, Dimitris A. Pados, State

University of New York at Buffalo, United States

TP8a1-7 Estimation of Subspace Occupancy

Kaitlyn Beaudet, Douglas Cochran, Arizona State

University, United States

TP8a1-8 Performance Analysis: DF Cognitive Network with

Transceiver Imperfections

Dang Khoa Nguyen, Kyushu Institute of Technology,

Japan; Tu Thanh Lam, Post and Telecommunications

Institute of Technology, Viet Nam; Hiroshi Ochi, Kyushu

Institute of Technology, Japan

\section{Session TP8a2 Signal Processing Methods}

Chair: Seung Jun Kim, University of Maryland, Baltimore County

$$
\text { 1:30 PM-3:10 PM }
$$

TP8a2-1 Blind Equalization Based On Blind Separation with Toeplitz Constraint

Zhengwei Wu, Saleem Kassam, University of

Pennsylvania, United States

TP8a2-2 Piecewise-Constant Recovery via Spike-and-Slab Approximate Message-Passing using a Scalarwise Denoiser

Jaewook Kang, Heung-No Lee, Kiseon Kim, Gwangju Institute of Science and Technology (GIST), Republic of Korea

TP8a2-3 Resource Allocation Optimization for Distributed Vector Estimation with Digital Transmission

Alireza Sani, Azadeh Vosoughi, University of Central Florida, United States

TP8a2-4 Exploiting the Cramér-Rao Bound for Optimised Sampling and Quantisation of FRI Signals Andre Angierski, Volker Kuehn, University of Rostock, Germany

TP8a2-5 Adaptive Waveform for Integrated Detection and Identification of Moving Extended Target Jo-Yen Nieh, Ric Romero, Naval Postgraduate School, United States

TP8a2-6 Channel Gain Cartography Via Low Rank and Sparsity Donghoon Lee, Seung-Jun Kim, University of Minnesota, United States

TP8a2-7 Bayesian Cramér-Rao Bound for Distributed Estimation of Correlated Data with Non-linear Observation Model Mojtaba Shirazi, Azadeh Vosoughi, University of Central Florida, United States

TP8a2-8 Multirate Processing Using Nested Sampling Peter Vouras, Naval Research Laboratory, United States 


\section{Session TP8a3 Image Processing II}

Chair: Ashkan Ashrafi, San Diego State University

1:30 PM-3:10 PM

TP8a3-1 Smoothed Rank Approximation Algorithms for Matrix Completion

Mohammed Al-Qizwini, Hayder Radha, Michigan State University, United States

TP8a3-2 Visibility Prediction of Flicker Distortions on Naturalistic Videos

Lark Kwon Choi, Lawrence Cormack, Alan Bovik, University of Texas at Austin, United States

TP8a3-3 Image Compression via Wavelets and Row Compression Mary HudachekBuswell, Georgia Institute of Technology, United States; Michael Stewart, Saied Belkasim, Georgia State University, United States

TP8a3-4 Low Complexity Dimensionality Reduction for Hyperspectral Images

Seda Senay, Hector Erives, New Mexico Institute of Mining and Technology, United States

TP8a3-5 Improving Image Clustering using Sparse Text and the Wisdom of the Crowds

Anna Ma, Claremont Graduate University, United States; Arjuna Flenner, Naval Air Warfare Center, United States; Deanna Needell, Claremont McKenna College, United States; Allon Percus, Claremont Graduate University, United States

TP8a3-6 Color Image Watermarking Using Quaternion Wavelets Lahouari Ghouti, King Fahd University of Petroleum and Minerals, Saudi Arabia

TP8a3-7 Immersion Ultrasonic Array Imaging Using a New Array Spatial Signature in Different Imaging Algorithms Nasim Moallemi, Shahram Shahbazpanahi, University of Ontario Institute of technology, Canada

TP8a3-8 A Proof on the Invariance of the Hirschman Uncertainty to the Rényi Entropy Parameter and an Observation on its Relevance in the Image Texture Classification Problem

Kirandeep Ghuman, Victor DeBrunner, Florida State University, United States

\section{Session TP8a4 Sensor and Wireless Networks}

Chair: Usman Khan, Tufts University

$$
\text { 1:30 PM-3:10 PM }
$$

TP8a4-1 Design of Orthogonal Golomb Rulers with Applications in Wireless Localization.

Omotayo Oshiga, Giuseppe Abreu, Jacobs University

Bremen, Germany 
TP8a4-2 Secrecy Outage Analysis of Cognitive Wireless Sensor

Networks

Satyanarayana Vuppala, Jacobs University Bremen, Germany; Weigang Liu, Tharmalingam Ratnarajah, University of Edinburgh, United Kingdom; Giuseppe Abreu, Jacobs University Bremen, Germany

TP8a4-3 On the Convergence Rate of Swap-Collide Algorithm for Simple Task Assignment

Sam Safavi, Usman A. Khan, Tufts University, United States

TP8a4-4 On the Impact of Low-Rank Interference on Distributed Multi-Agent Optimization

Chenguang Xi, Usman A. Khan, Tufts University, United States

TP8a4-5 Multipath-Aided Cooperative Network Localization Using Convex Optimization

Hassan Naseri, Mario Pereira da Costa, Visa Koivunen, Aalto University, Finland

TP8a4-6 Mobile Sensor Mapping via Semi-Definite Programming Giuseppe Destino, Davide Macagnano, University of Oulu, Finland

TP8a4-7 Indoor Node Localization using Geometric Dilution of Precision in Ad-Hoc Sensor Networks

Sudhir Kumar, Rajesh M. Hegde, Indian Institute of Technology Kanpur, India

TP8a4-8 Efficient Consensus Synchronization via Implicit Acknowledgment Andrew G. Klein, Western Washington University, United States; D. Richard Brown III, Worcester Polytechnic Institute, United States

\section{Session TP8b1 Topics in Communication Systems}

Chair: Alexios Balatsoukas-Stimming, EPFL

$$
\text { 3:30 PM-5:10 PM }
$$

TP8b1-1 Performance Analysis of a MMSE Turbo Equalizer with LDPC in a FTN Channel with Application to Digital Video Broadcast

Ghassan Maalouli, Brian A. Banister, Comtech EF Data, United States

TP8b1-2 Characteristics of Optical Scattering and Turbulence Communication Channels

Weihao Liu, Zhengyuan Xu, University of Science and Technology of China, China

TP8b1-3 Comparison of SNR and Peak-SNR (PSNR) Performance Measures and Signals for Peak-limited Two-Dimensional (2D) Pixelated Optical Wireless Communication

Eyal Katz, Yeheskel Bar-Ness, New Jersey Institute of Technology, United States

TP8b1-4 I.I.D. Stochastic Analysis of PWM Signals Noyan Sevuktekin, Andrew Singer, University of Illinois at Urbana-Champaign, United States 
TP8b1-5 Statistical Data Correction for Unreliable Memories Christoph Roth, ETH-Zurich, Switzerland; Christoph Struder, Cornell University, United States; Georgios Karakonstantis, Andreas Burg, École Polytechnique Fédérale de Lausanne, Switzerland

TP8b1-6 Sonar Data Compression using Non-Uniform Quantization and Noise Shaping Lok Wong, Gregory Allen, Brian Evans, University of Texas at Austin, United States

TP8b1-7 Multilevel Coding for Non-Orthogonal Broadcast Stephan Pfletschinger, Monica Navarro, Centre Tecnologic de Telecomunicacions de Catalunya, Spain; Christian Ibars, Intel Corporation, United States

TP8b1-8 Dynamic Target Identification and Classification Based on Resonance Topography Grouping Ananya Sen Gupta, Daniel Schupp, University of Iowa, United States; Ivars Kirsteins, Naval Undersea Warfare Center, United States

\section{Session TP8b2 Relays, Cognitive, Cooperative, and Heterogeneous Networks}

Chair: Andrew G. Klein, Worcester Polytechnic Institute 3:30 PM-5:10 PM

TP8b2-1 A Distributed Algorithm for Energy Saving in Nomadic Relaying Networks

Zhe Ren, BMW Group Research and Technology, Germany; Mahdy Shabeeb, Munich University of Technology, Germany; Slawomir Stanczak, Fraunhofer Institute for Telecommunications Heinrich Hertz Institute, Germany; Peter Fertl, BMW Group Research and Technology, Germany

TP8b2-2 Instantaneous Relaying for the 3-Way Relay Channel with Circular Message Exchanges

Bho Matthiesen, Eduard A. Jorswieck, Technische Universität Dresden, Germany

TP8b2-3 On the Performance of Hybrid Satellite-Terrestrial Cooperative Networks with Interferences Min Lin, PLA University of Science and Technology, China; Jian Ouyang, Nanjing University of Posts and Telecommunications, China; Zhu Wei-Ping, Concordia University, Canada

TP8b2-4 An Online Parallel Algorithm for Spectrum Sensing in Cognitive Radio Networks

Yang Yang, Technische Universitaet Darmstadt, Germany; Mengyi Zhang, Chinese University of Hong Kong, Hong Kong SAR of China; Marius Pesavento, Technische Universitaet Darmstadt, Germany; Daniel Palomar, Hong Kong University of Science and Technology, Hong Kong SAR of China 
TP8b2-5 On the Spatial Spectral Efficiency of ITLinQ

Ratheesh Mungara, Universitat Pompeu Fabra, Spain;

Xinchen Zhang, University of Texas at Austin, United

States; Angel Lozano, Universitat Pompeu Fabra, Spain;

Robert W. Heath Jr., University of Texas at Austin, United

States

TP8b2-6 Time and Frequency Self-Synchronization in Dense

Cooperative Networks

Maria Antonieta Alvarez, Bahar Azari, Umberto

Spagnolini, Politecnico di Milano, Italy

TP8b2-7 Effect of Cluster Rotation Speed in Coordinated

Heterogeneous MIMO Cellular Networks with

Proportionally Fair User Scheduling

Hakimeh Purmehdi, Robert Elliott, Witold Krzymien,

University of Alberta, Canada; Jordan Melzer, TELUS

Communications, Canada

TP8b2-8 Relay Selection for AF Wireless Relay Networks in Adverse Communication Environments

Kanghee Lee, Republic of Korea Air Force, Republic of Korea; Visvakumar Aravinthan, Sunghoon Moon,

Wichita State University, United States; Jongbum Ryou,

Changki Moon, Inha Hyun, Republic of Korea Air Force,

Republic of Korea; Sun Jo, Defense Acquisition Program Administrtion of ROK, Republic of Korea

\section{Session TP8b3 Signal Processing Architectures}

Chair: Zain Ul-Abdin, Halmstad University

3:30 PM-5:10 PM

TP8b3-1 Hybrid Floating-Point Modules with Low Area Overhead on a Fine-Grained Processing Core

Jon Pimentel, Bevan Baas, University of California,

Davis, United States

TP8b3-2 Scalable Hardware-Based Power Management for ManyCore Systems

Bin Liu, Brent Bohnenstiehl, Bevan Baas, University of California, Davis, United States

TP8b3-3 Optimized FPGA Based Implementation of Discrete Wavelet Transform

Amin Jarrah, Mohsin M. Jamali, University of Toledo, United States

TP8b3-4 Mapping and Scheduling of Dataflow Graphs - A Systematic Map

Usman Mazhar Mirza, Mehmet Ali Arslan, Gustav

Cedersjö, Sardar Muhammad Sulaman, Jörn W. Janneck,

Lund University, Sweden

TP8b3-5 Dataflow Machines

Jörn W. Janneck, Gustav Cedersjö, Lund University,

Sweden; Endri Bezati, Simone Casale Brunet, École

Polytechnique Fédérale de Lausanne, Switzerland

TP8b3-6 Replacement Techniques for Improving Performance in Sub-Block Caches

Oluleye Olorode, Mehrdad Nourani, University of Texas at Dallas, United States 
TP8b3-7 Dynamic Reconfiguration of FPGA-based Multi-

Processor Arrays

James Glenn-Anderson, Supercomputer Systems, Inc.,

United States

TP8b3-8 Coprime Processing for the Elba Island Sonar Data Set

Vaibhav Chavali, Kathleen Wage, George Mason

University, United States; John Buck, University of

Massachusetts Dartmouth, United States

\section{Session TP8b4 Signal Processing Theory and Applications}

Chair: Yue Lu, Harvard University

\section{3:30 PM-5:10 PM}

TP8b4-1 Prediction of a Bed-Exit Motion: Multi-Modal Sensing Approach and Incorporation of Biomechanical Knowledge Jun Hao, Xiaoxiao Dai, Amy Stroder, Jun Zhang, Bradley Davidson, Mohammad Mahoor, University of Denver, United States; Neil McClure, OKT Enterprises, United States

TP8b4-2 Ultra-Wideband Radar based Human Body Landmark Detection and Tracking with Biomedical Constraints for Human Motion Measuring

Xiaoxiao Dai, Zhichong Zhou, Jun Zhang, Bradley Davidson, University of Denver, United States

TP8b4-3 Separation of Interleaved Markov Chains Ariana Minot, Yue Lu, Harvard University, United States

TP8b4-4 Ramanujan Subspaces and Digital Signal Processing P. P. Vaidyanathan, California Institute of Technology, United States

TP8b4-5 Asynchronous Discrete-time Signal Processing with Molecular Reactions

Sayed Ahmad Salehi, Marc Riedel, Keshab K. Parhi, University of Minnesota, United States

TP8b4-6 Sequential Prediction of Individual Sequences in the Presence of Computational Errors

Mehmet Donmez, Andrew Singer, University of Illinois at Urbana Champaign, United States

TP8b4-7 A Scalable Feature Learning and Tag Prediction Framework for Natural Environment Sounds Prasanna Sattigeri, Arizona State University, United States; Jayaraman Thiagarajan, Lawrence Livermore National Laboratory, United States; Mohit Shah, Arizona State University, United States; Karthikeyan Ramamurthy, IBM Research, United States; Andreas Spanias, Arizona State University, United States

TP8b4-8 Extending Coherence for Optimal Detection of Nonstationary Harmonic Signals Scott Wisdom, University of Washington, United States; James Pitton, Applied Physics Laboratory and University of Washington, United States; Les Atlas, University of Washington, United States 


\section{Session WA1a MIMO Design for mmWave}

\section{Systems}

Chair: Zhouyue Pi, Samsung

WA1a-1 A Tractable Model for Rate in Noise Limited 8:15 AM mmWave Cellular Networks

Sarabjot Singh, Mandar Kulkarni, Jeffrey Andrews,

University of Texas at Austin, United States

WA1a-2 MIMO Designs for mmWave Wireless LAN 8:40 AM Systems

Sridhar Rajagopal, Shadi Abu-Surra, Sudhir

Ramakrishna, Rakesh Taori, Samsung Research America, United States

WA1a-3 Analysis of Millimeter Wave Cellular 9:05 AM

Networks with Overlaid Microwave Base Stations

Tianyang Bai, Robert W. Heath Jr., University of Texas at Austin, United States

WA1a-4 Initial Beamforming for mmWave 9:30 AM Communications

Vip Desai, Philippe Sartori, Weimin Xiao, Anthony Soong, Lukasz Krzymien, Huawei Technologies Co., Ltd., United

States; Ahmed Alkhateeb, University of Texas at Austin,

United States

\section{Session WA1b Massive MIMO II}

Chair: David J. Love, Purdue University

WA1b-1 A Multistage Linear Receiver Approach for 10:15 AM MMSE Detection in Massive MIMO

Ting Li, Sujeet Patole, Murat Torlak, University of Texas at Dallas, United States

WA1b-2 Beamforming-Based Spatial Precoding in 10:40 AM FDD Massive MIMO Systems

Ming-Fu Tang, Meng-Ying Lee, Borching Su, National Taiwan University, Taiwan; Chia-Pang Yen, Industrial Technology Research Institute, Taiwan

WA1b-3 Asymmetric Distributed Space Frequency 11:05 AM Coded Cooperative Network for Large Scale MIMO Bhagyashri Honrao, Chirag Warty, Shikha Nema, SNDT University, India

\section{Session WA2a 5G and Energy Efficient Cellular Networks}

Chair: Jinkang Zhu, University of Science and Technology of China

WA2a-1 Traffic Aware Offloading for BS Sleeping in 8:15 AM Heterogeneous Networks

Shan Zhang, Sheng Zhou, Zhisheng Niu, Tsinghua

University, China

WA2a-2 A Survey on 5G New Waveform: From Energy Efficiency Aspects

Shunqing Zhang, Xiuqiang Xu, Yiqun Wu, Lei Lu, Yan

Chen, Huawei Technologies Co., Ltd., China 
WA2a-3 Evolution of LTE and new Radio Access

9:05 AM

Technologies for FRA (Future Radio Access)

Hidetoshi Kayama, Huiling Jiang, DOCOMO Beijing

Communications Laboratories Co. Ltd., China

WA2a-4 A Novel Cell-Interference Model and

9:30 AM

Performance Analysis of the Future Wireless

Networks

Jinkang Zhu, Haibao Ren, University of Science and

Technology of China, China

\section{Session WA2b Mobile Health}

Chair: Mi Zhang, Cornell University

WA2b-1 On Outlier Detection in R-R Intervals from 10:15 AM

ECG Data Collected in the Natural Field

Environment

Rummana Bari, Santosh Kumar, University of Memphis,

United States

WA2b-2 Patient-Centric On-Body Sensor Localization 10:40 AM in Smart Health Systems

Ramyar Saeedi, Hassan Ghasemzadeh, Washington State University, United States

WA2b-3 Making Sense of Personal Data in Clinical 11:05 AM Settings

Harinath Garudadri, University of California, San Diego,

United States

\section{Session WA3a Sparse Learning and Estimation}

Chair: Ali Pezeshki, Colorado State University

WA3a-1 Sparse Bayesian Learning Using Approximate 8:15 AM

Message Passing

Maher Al-Shoukairi, Bhaskar Rao, University of

California, San Diego, United States

WA3a-2 Hierarchical Bayesian Approach for

Jointly-Sparse Solution of Multiple-Measurement

Vectors

Mohammad Shekaramiz, Todd K. Moon, Jacob H.

Gunther, Information Dynamics Laboratory / Utah State

University, United States

WA3a-3 Dictionary Approaches For Identifying 9:05 AM

Periodicities in Data

Srikanth Venkata Tenneti, P. P Vaidyanathan, California Institute of Technology, United States

WA3a-4 An Asymptotic Maximum Likelihood 9:30 AM

Estimator for the Period of a Cyclostationary

Process

David Ramirez, Peter J. Schreier, University of Paderborn,

Germany; Javier Via, Ignacio Santamaría, University

of Cantabria, Spain; Louis L. Scharf, Colorado State

University, United States 


\section{Session WA3b Advances in Statistical Learning}

Chair: Kobi Cohen, University of Illinois at Urbana-Champaign

WA3b-1 Quasicontinuous State Hidden Markov

10:15 AM

Models Incorporating State Histories

Todd K. Moon, Jacob H. Gunther, Utah State University,

United States

WA3b-2 A Classification Centric Quantizer for 10:40 AM Efficient Encoding of Predictive Feature Errors

Scott Deeann Chen, Pierre Moulin, University of Illinois at Urbana-Champaign, United States

WA3b-3 Time-Varying Stochastic Multi-Armed Bandit 11:05 AM Sattar Vakili, Qing Zhao, Yuan Zhou, University of

California, Davis, United States

\section{Session WA4a Physical Layer Security II}

Chair: Pin-Hsun Lin, TU Dresden

WA4a-1 Investigation of Secure Wireless Regions

$8: 15$ AM

Using Configurable Beamforming on WARP

platform

Yuanrui Zhang, Queen's University Belfast, United

Kingdom; Bei Yin, Rice University, United States; Roger

Woods, Queen's University Belfast, United Kingdom;

Joseph R. Cavallaro, Rice University, United States;

Alan Marshall, University of Liverpool, United Kingdom;

Youngwook Ko, Queen's University Belfast, United

Kingdom

WA4a-2 Wiretap-Channels with Constrained Active $\quad$ 8:40 AM

Attacks

Carsten Rudolf Janda, Christian Scheunert, Eduard A.

Jorswieck, Dresden University of Technology, Germany

WA4a-3 Secrecy Rate Maximization for Information 9:05 AM and Energy Transfer in MIMO Beamforming

Networks

Jens Steinwandt, Ilmenau University of Technology,

Germany; Sergiy Vorobyov, Aalto University, Finland;

Martin Haardt, Ilmenau University of Technology,

Germany

WA4a-4 Everlasting Secrecy in Disadvantaged

Wireless Environments against Sophisticated

Eavesdroppers

Azadeh Sheikholeslami, Dennis Goeckel, Hossein Pishro-

nik, UMASS-Amherst, United States

\section{Session WA4b Coding and Decoding}

Chair: James A. Ritcey, University of Washington

WA4b-1 Noisy Belief Propagation Decoder

10:15 AM

Chu-Hsiang Huang, Yao Li, Lara Dolecek, University of

California, Los Angeles, United States

WA4b-2 A Low-Complexity Improved Successive 10:40 AM Cancellation Decoder for Polar Codes

Orion Afisiadis, Alexios Balatsoukas-Stimming, Andreas

Burg, École Polytechnique Fédérale de Lausanne,

Switzerland 
State Dependent Mappings

Ruey-Yi Wei, National Central University, Taiwan; James

Ritcey, University of Washington, United States

\section{Session WA5a Information Processing for Social and Sensor Networks}

Chair: Michael Rabbat, McGill University

WA5a-1 Fourier Transform for Signals on Dynamic $\quad$ 8:15 AM

Graphs

Arash Golibagh Mahyari, Selin Aviyente, Michigan State

University, United States

WA5a-2 Anomalous Subgraph Detection in

Publication Networks: Leveraging Truth

Nadya Bliss, Manfred Laubichler, Arizona State

University, United States

WA5a-3 Identifying Congestion in Software-Defined 9:05 AM

Networks

Thomas Parker, Jamie Johnson, Murali Tummala, John

McEachen, James Scrofani, Naval Postgraduate School,

United States

WA5a-4 Vulnerability of CPS inference to DoS attacks 9:30 AM Mohammadreza Doostmohammadian, Usman A. Khan,

Tufts University, United States

\section{Session WA5b Document Processing and Synchronization}

Chair: Olgica Milenkovic, University of Illinois at Urbana-

Champaign

WA5b-1 Synchronizing Ordinal Data over Noisy $\quad$ 10:15 AM Channels

Han Mao Kiah, Lili Su, Olgica Milenkovic, University of Illinois at Urbana-Champaign, United States

WA5b-2 Efficient Synchronization of Files in 10:40 AM Distributed Storage Systems

Salim El Rouayheb, Illinois Institute of Technology, United

States; Sreechakra Goparaju, Princeton University, United States; Han Mao Kiah, Olgica Milenkovic, University of Illinois at Urbana-Champaign, United States

WA5b-3 Efficient File Synchronization: Extensions 11:05 AM and Simulations

Clayton Schoeny, Nicolas Bitouze, Frederic Sala, Lara Dolecek, University of California, Los Angeles, United States 


\section{Session WA6a Adaptive Signal Design and}

\section{Analysis}

Chair: Antonia Papandreou-Suppappola, Arizona State University

WA6a-1 Eigen-Basis Analysis of Expected Cumulative 8:15 AM

Modulus for Constrained Signal Design

Aaron Jones, Air Force Research Laboratory, United

States; Brian Rigling, Wright State University, United

States; Muralidhar Rangaswamy, Air Force Research

Laboratory, United States

WA6a-2 Characterization of Information in Phase of 8:40 AM

Radar Range Profiles

Linda Moore, Air Force Research Laboratory / University

of Dayton, United States; Brian Rigling, Wright State

University, United States; Robert Penno, University of

Dayton, United States

WA6a-3 Radar Tracking Waveform Design in

Continuous Space and Optimization Selection

Using Differential Evolution

Antonia Papandreou-Suppappola, Bryan Paul, Daniel

Bliss, Arizona State University, United States

WA6a-4 Reduced Rank Adaptive Filtering in 9:30 AM Impulsive Noise Environments

Hamza Soury, King Abdullah University of Science and Technology (KAUST), Saudi Arabia; Karim Abed-Meraim, Polytech Orleans, France; Mohamed-Slim Alouini, King Abdullah University of Science and Technology (KAUST), Saudi Arabia

\section{Session WA6b Distributed Detection and Optimization}

Chair: Andrea Simonetto, Delft University of Technology

WA6b-1 Distributed Detection for Wireless Sensor 10:15 AM

Networks with Fusion Center under Correlated

Noise

Alireza S. Behbahani, Ahmed M. Eltawil, Hamid

Jafarkhani, University of California, Irvine, United States

WA6b-2 Distributed Asynchronous Time-Varying 10:40 AM

Constrained Optimization

Andrea Simonetto, Geert Leus, Delft University of

Technology, Netherlands

WA6b-3 M-ary Distributed Detection in the Presence 11:05 AM of Channel Estimation Error

Zahra Hajibabaei, Azadeh Vosoughi, University of Central Florida, United States

\section{Session WA7a Implementation of Wireless Systems}

Chair: Roger Woods, Queens University

WA7a-1 Field-Order Based Hardware Cost Analysis of 8:15 AM Non-Binary LDPC Decoders

Yuta Toriyama, Behzad Amiri, Lara Dolecek, Dejan

Markovic, University of California, Los Angeles, United

States 
WA7a-2 Algorithm and Architecture for Hybrid

Decoding of Polar Codes

Bo Yuan, Keshab K. Parhi, University of Minnesota, Twin Cities, United States

WA7a-3 A Signal Processing Approach Towards

9:05 AM

Ultra-Low Power Transceiver Design

Vijay Venkateswaran, Pawel Rulikowski, Howard Huang,

Bell Labs, Ireland

WA7a-4 A High Performance GPU-based

9:30 AM

Software-defined Basestation

Kaipeng Li, Michael Wu, Guohui Wang, Joseph R.

Cavallaro, Rice University, United States

\section{Session WA7b Video Coding Architecture and Design}

Chair: Jorn Janneck, Lund University

WA7b-1 Development and Optimization of High Level 10:15 AM

Dataflow Programs: the HEVC Decoder Design

Case

Khaled Jerbi, INSA of Rennes / IETR, France; Daniele

Renzi, Damien De Saint-Jorre, École Polytechnique

Fédérale de Lausanne, Switzerland; Hervé Yviquel,

INSA of Rennes / IETR, France; Claudio Alberti, École

Polytechnique Fédérale de Lausanne, Switzerland;

Mickaël Raulet, INSA of Rennes / IETR, France; Marco

Mattavelli, École Polytechnique Fédérale de Lausanne,

Switzerland

WA7b-2 A Low-Power Hybrid Video Recording 10:40 AM

System with H.264/AVC and Light-Weight

Compression

Hyun Kim, Seoul National University, Republic of Korea;

Chae Eun Rhee, Inha University, Republic of Korea;

Hyuk-Jae Lee, Seoul National University, Republic of

Korea

WA7b-3 Design of View Synthesis Prediction in

11:05 AM

3D-HEVC via Algorithmic Complexity Analysis

Gwo Giun (Chris) Lee, Bo-Syun Li, Chun-Fu Chen,

National Cheng Kung University, Taiwan 



\section{Author List}

NAME

Aazhang, Behnaam

Abed-Meraim, Karim..

Abramovich, Yuri

Abreu, Giuseppe

Abreu, Giuseppe

Abreu, Giuseppe

Abry, Patrice .

Abu-Surra, Shadi

Acton, Scott

Adalbjörnsson, Stefan Ingi...

Adhikary, Ansuman .

Afisiadis, Orion

Aghagolzadeh, Mohammad.

Aguiar, Pedro

Ahmad, Fauzia

Ahmad, Fauzia

Ahmad, Waquar.

Ahmadi, Seyed-Ahmad

Ahmed, Rameez

Aiello, Katherine

Aiello, Katherine

Akcakaya, Murat

Alberti, Claudio

Aldhahab, Ahmed

Al-Dhahir, Naofal

Alkhateeb, Ahmed

Alkhateeb, Ahmed

Allen, Gregory.

Alouini, Mohamed-Slim.

Alqadah, Hatim

Al-Qizwini, Mohammed.

Al-Saggaf, Ubaid

Alshamary, Haider

Al-Shoukairi, Maher

Alter, Orly

Alter, Orly

Alter, Orly.

Alvarez, Maria Antonieta.

Amari, Abdelkerim

Amin, Moeness

Amin, Moeness

Amiri, Behzad .

Amiri Eliasi, Parisa

An, Kang.

Anderson, John

Andrade, Joao.

Andrews, Jeffrey

Angierski, Andre

Anticevic, Alan

Anttila, Lauri.....

Aravinthan, Visvakumar.

\section{SESSION NAME}

..TA8a3-5 Aravinthan, Visvakumar...

WA6a-4

..TA7b-1

...TA8b3-6

. TP8a4-1

. TP8a4-2

TA5b-4

WA1a-2

MA5b-1

TA8b4-4

MP4b-1

WA4b-2

...MP7b-3

..TA6b-3

..TA6a-3

...TA7b-4

..TA5a-1

MP2b-2

.MP4a-2

.MP8a2-1

.MP8a2-2

.MA2b-4

WA7b-1

TA8a2-4

TA8a3-6

..TA4a-3

WA1a-4

.. TP8b1-6

WA6a-4

.MA8b3-7

.. TP8a3-1

... TA8a4-4

...TA8a1-2

WA3a-1

.MP8a2-1

.MP8a2-2

..TA1b-2

.. TP8b2-6

TP4a-1

..TA6a-3

...TA7b-4

WA7a-1

.MP8a2-6

.MA8b2-1

..TA7b-3

..MP8a4-2

WA1a-1

.. TP8a2-4

..TA2b-1

...TA8a1-5

MA8b2-5

Arbabian, Amin .

Arge, Charles.
Argyropoulos, Paraskevas...........MP8a4-5

Arikan, Orhan ...............................MP3b-3

Arikan, Orhan ..............................TA8b4-5

Arslan, Mehmet Ali...................... TP8b3-4

Asad, Syed .................................TA8a4-6

Asghari, Mohammad H................ TA8a2-3

Ashrafi, Ashkan..............................TA5a-4

Astely, David ..................................TA4a-2

Athanas, Peter............................ TP7a-4

Atia, George .............................MA8b4-6

Atia, George ..................................MP3b-2

Atia, George ..............................TA8a2-4

Atia, George .................................TA8b4-7

Atlas, Les.................................. TP5a-4

Atlas, Les................................... TP8b4-8

Aviyente, Selin ...........................MA8b4-5

Aviyente, Selin .............................MP8a5-5

Aviyente, Selin ............................. WA5a-1

Azari, Bahar................................. TP8b2-6

Azizyan, Martin .............................MP3a-3

Ba, Demba..................................TA2a-1

Baas, Bevan ................................ TP8b3-1

Baas, Bevan ………................ TP8b3-2

Babadi, Behtash ….......................TA2a-1

Babu, Prabhu............................... TP3b-2

Badreldin, Islam............................TA2a-4

Bai, Tianyang...............................WA1a-3

Bajwa, Waheed ..............................TA6a-2

Balatsoukas-Stimming, Alexios ... WA4b-2

Banister, Brian A ........................ TP8b1-1

Bardak, Burak ........................... TP7a-3

Bari, Mohammad .........................TA8a3-2

Bari, Rummana..............................WA2b-1

Bar-Ness, Yeheskel ...................... TP4a-4

Bar-Ness, Yeheskel ................... TP8b1-3

Bartels, Randy .............................MP3a-4

Basiri, Shahab ...............................MA1b-2

Basten, Twan ..............................MP7a-2

Basu, Prabahan ...........................TA8b1-4

Batalama, Stella N...................... TP8a1-6

Beaudet, Kaitlyn ......................... TP8a1-7

Behbahani, Alireza S. ................... WA6b-1

Behgam, Mohammad .................. TP2b-1

Belkasim, Saied.......................... TP8a3-3

Bell, Kristine................................ TP6a-3

Bell, Mark R..............................MA8b3-4

Benesty, Jacob ............................ TP2b-3

Benesty, Jacob .......................... TP2b-4 


\section{NAME}

Benetti, Michele

Bently, Edward.

Berardinelli, Gilberto

Berberidis, Dimitrios

Bezati, Endri.....

Bhaskar, Badri

Bhattacharyya, Shuvra

Bhorkar, Abhijeet

Billings, Jacob.

bin Mansoor, Umair

Bingman, Verner

Biswal, Bharat

Biswas, Sampurna

Bitouze, Nicolas.

Bliss, Daniel...

Bliss, Daniel.

Bliss, Daniel......

Bliss, Nadya

Bo Jensen, Nicklas

Bohnenstiehl, Brent

Bolic, Miodrag

Bolucek, Muhsin Alperen ............MP8a4-7

Bonnichsen, Lars.

Borisch, Eric

Bourennane, Salah.

Bovik, Alan.

Bovik, Alan.

Brahma, Swastik

Brandt-Pearce, Maite.

Brisk, Philip.

Brock-Nannestad, Laust.

Brooks, Dana H.

Brorsson, Mats

Brown, Christopher.

Brown, Donald

Brown, Emery.

Brown, Matthew.

Brown III, D. Richard

Brown III, D. Richard

Bruck, Jehoshua

Brumberg, Jonathan

Brynolfsson, Johan.

Buck, John.

Buck, John.

Bucklew, James.

Burg, Andreas.

Burg, Andreas.

Burg, Andreas.

Burgess, Neil

Burnison, Jeremy.

Burton, Andrew

Buthler, Jakob L

Cadambe, Viveck

Caire, Giuseppe.
SESSION

TA8a2-5

TP7b-4

.MP8a4-6

MA1b-4

TP8b3-5

MP3a-1

MP7a-1

TA8b1-7

.MP8a2-4

TA8a4-6

MP8a5-3

TA2b-4

...MP3b-4

WA5b-3

MA8b4-4

TP5b-3

WA6a-3

WA5a-2

TP7a-2

TP8b3-2

MP6b-4

TP7a-2

MP8a2-3

..MP1b-2

MA5b-3

TP8a3-2

TP6a-4

TP7b-1

...MP7a-4

TP7a-2

MA2b-4

TP6b-1

...TA5b-3

..MP8a1-3 ..TA2a-1

..TA7b-2

TA8a1-6

TP8a4-8

TP2a-1

MA2b-2

TA8b4-8

..MA8b3-2

TP8b3-8

MP8a2-5

MP8a4-2

TP8b1-5

WA4b-2

TA7a-3

MA2b-2

TP7b-4

MP8a4-6

.MP8a2-7

MP4b-1
NAME

SESSION

Calderbank, Robert .........................TA6a-2

Calhoun, Vince ...............................TA2b-3

Campagnaro, Filippo …..................MA3b-1

Cao, Nianxia ................................. TP6a-4

Casale Brunet, Simone............... TP8b3-5

Casari, Paolo .................................MA3b-1

Casas, Christian Ibars .................TA8b1-7

Castedo, Luis...............................TA8b1-1

Castrillon, Gabriel ..........................MP2b-2

Castro-Arvizu, Juan Manuel ..........MP6b-3

Catbas, Necati ............................TA8b4-7

Caulfield, John ..............................MA5b-2

Cavallaro, Joseph R. ...................MP8a4-1

Cavallaro, Joseph R. ..................MP8a4-2

Cavallaro, Joseph R. .................. WA4a-1

Cavallaro, Joseph R. ................... WA7a-4

Cedersjö, Gustav............................ TP7a-1

Cedersjö, Gustav......................... TP8b3-4

Cedersjö, Gustav........................ TP8b3-5

Champagne, Benoit....................... TP5a-1

Chang, Yueh-Lun..........................MP5b-2

Chan-Tin, Eric.............................. TP8a1-5

Chavali, Vaibhav......................... TP8b3-8

Che, Tiben ...................................MA7b-4

Chen, Chien-Min...........................TA8a1-4

Chen, Chun-Fu ............................. WA7b-3

Chen, Jia ...................................... TP1b-1

Chen, Jianshu.................................MP5a-2

Chen, Jianshu................................TA6b-4

Chen, Jie .......................................TA1a-2

Chen, Jingdong …....................... TP2b-4

Chen, Scott Deeann .................... WA3b-2

Chen, Yan ...................................... WA2a-2

Chen, Yang......................................TA6b-2

Chen, Yejian .................................MA8b1-3

Cheney, Margaret .........................MA8b3-6

Cheng, Qi ..................................TA8b3-5

Cheng, Qi ................................. TP8a1-5

Cheng, Xiang.................................MP4a-4

Cheng, Xilin ...................................MP4a-4

Chepuri, Sundeep Prabhakar ....... TP3b-1

Chiba, Hironobu............................... TA5a-3

Chin, Sang (Peter).........................MA6b-3

Chitre, Mandar ...............................MA3b-2

Chklovskii, Dmitri ............................MP2b-3

Chklovskii, Dmitri ............................TA2a-2

Cho, Myung ................................TA8b4-1

Chockalingam, AnanthanarayananTA3b-3

Choi, Gwan ...................................MA7b-4

Choi, Gwan..................................TA8a3-4

Choi, Inyong ..................................... 2 2b-1

Choi, Junil.................................... TA3b-2

Choi, Lark Kwon .......................... TP8a3-2

Choi, Yang-Seok............................ TP5b-1 


\section{NAME}

Christensen, Mads Græsbøll......MP8a5-4

Christensen, Mads Græsbøll........ TP5a-3

Chua, Gabriel ......

MA3b-2

Ciblat, Philippe

TP4a-1

Ciochina, Silviu

TP2b-3

Closas, Pau

MP6b-3

Cochran, Douglas

Cochran, Douglas.

Cochran, Douglas.

Codreanu, Marian.

Cohen, Kobi...

Cole, Michael

Cormack, Lawrence.

Corr, Jamie MP8a3-7

TA8b3-7

TP8a1-7

TA8b4-6

. TP8a1-2

...TA2b-1

TP8a3-2

MP8a3-8

Cosman, Pamela.

...MP5b-2

Cosman, Pamela

TA8a2-2

Cottatellucci, Laura.

MP4b-2

Couillet, Romain

Coulon, Martial

TP1a-2

MP6b-1

Cousseau, Juan.

Creusere, Charles

Creusere, Charles

Crider, Lauren.

Cui, Guolong.

Curran, Tim.

Dabin, Jason.

Dahlman, Erik.

Dai, Xiaoxiao

Dai, Xiaoxiao

Dang, Chinh..

Dang, Wenbing

Dao, Minh

Dao, Minh

Dardari, Davide

Darsena, Donatella.

Dasgupta, Soura

Dauphin, Stephen.

Davidson, Bradley

Davidson, Bradley

Davis, Philip.

Davis, Philip...

Dawson, Martin...

De Carvalho, Elisabeth.

de Kerret, Paul.

de Sa, Virginia

De Saint-Jorre, Damien

DeBrunner, Linda S.

DeBrunner, Victor.

DeBrunner, Victor

DeBrunner, Victor

Declercq, David

Dehghannasiri, Roozbeh

Del Galdo, Giovanni

Demirors, Emrecan.
.MA8b2-6

.MA8b4-1

.MA8b4-2

.MP8a3-7

TP6a-2

..MA2b-3

...MP6b-1

..TA4a-2

.. TP8b4-1

TP8b4-2

TA8a2-6

..MP3a-4

MA6b-3

...TA6a-4

MP6b-2

...TA6b-1

...MP3b-4

.MA8b3-6

TP8b4-1

.. TP8b4-2

.MA8b4-1

.MA8b4-2

... TP7b-2

TA8a3-8

..TA4b-1

..MA2b-3

WA7b-1

TA8b2-7

TA8b2-7

TA8b4-3

TP8a3-8

MA7b-3

MP5b-4

TA8b4-5

TP8a1-6

\section{NAME}

Desai, Vip

SESSION

Destino, Giuseppe ...................... TP8a4-6

Dick, Christopher ..........................MP8a4-1

Dick, Christopher ......................... TP5b-4

Ding, Eric Wei-Jhong .....................MP6a-3

Djuric, Petar .........................................

Do, Anh.......................................TA5b-3

Dogandžić, Aleksandar................ TP3b-4

Dolecek, Lara ............................. WA4b-1

Dolecek, Lara ................................WA5b-3

Dolecek, Lara ................................WA7a-1

Donmez, Mehmet ........................ TP8b4-6

Doostmohammadian, Mohammadreza ....

WA5a-4

Doostnejad, Roya .......................... TP5b-1

Doroslovacki, Milos.......................TA8a3-2

Doroslovacki, Milos......................TA8a4-2

Doty, David ………...................... TP2a-4

Douglas, Scott ..............................MP6a-4

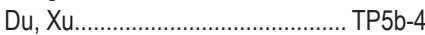

Duffy, Ken .................................MP8a2-7

Dupret, Antoine .............................TA8a2-5

Dutta, Arindam ..............................MA8b4-7

Edfors, Ove ...................................MP4b-4

El Rouayheb, Salim ...................... WA5b-2

Elgala, Hany …............................... TP7b-3

El-Keyi, Amr .................................A8b2-4

Elliott, Robert............................. TP8b2-7

Eltawil, Ahmed M......................... WA6b-1

Enzner, Gerald............................... TP2b-2

Ercegovac, Milos ........................TA8b2-4

Erdinc, Ozgur..............................TA8a4-7

Erdogan, Alper Tunga...................MP8a3-2

Erdogmus, Deniz ...........................MA2b-4

Erives, Hector .............................. TP8a3-4

Eslami Rasekh, Maryam..............MA8b1-1

Evans, Brian .................................MP5b-1

Evans, Brian ................................ TA8a2-8

Evans, Brian ............................... TP8b1-6

Facchinei, Francisco.......................MA1b-1

Falcao, Gabriel ...........................MP8a4-2

Falk, Joachim ..............................MP7a-2

Falk, Tiago ...................................MP2a-1

Fan, Guoliang .............................MA5b-2

Farnoud, Farzad ............................. TP2a-1

Farnoud, Farzad ........................... TP2a-3

Favaro, Federico ...........................MA3b-1

Feng, Li....................................MP8a2-6

Ferdinand, Nuwan ........................TA8a3-5

Fernandez-Canellas, Delia ...........MA2b-4

Fernández-Rubio, Juan .................MP6b-3

Ferrari, André................................. TA3a-2

Fertl, Peter................................... TP8b2-1

Fijalkow, Inbar..............................TA8a2-1 


\section{NAME}

Filippou, Miltiades

Firouzbakht, Koorosh

Fischione, Carlo.

Flenner, Arjuna

Ford, Russell

Forsell, Martti

Fortin, Benoit

Frazer, Gordon

Friedlander, Benjamin

Friedlander, Benjamin

Friedlander, Benjamin.

Frølund Pedersen, Gert

Fruth, Frank

Fry, Alexandra.

Gangadharan, Deepak

Gao, David Wenzhong

Gao, David Wenzhong

Gao, Xiang.

Garcia, Nil

Garudadri, Harinath

Geilen, Marc ....

Gelli, Giacinto

Georgescu, Ramona .

Gerges, Ramez L.

Gesbert, David.

Gesbert, David.

Ghadimi, Euhanna....

Ghadiyaram, Deepti.

Ghasemzadeh, Hassan

Ghassemlooy, Z.

Ghods, Alireza

Ghouti, Lahouari.

Ghuman, Kirandeep

Giannakis, Georgios

Giannakis, Georgios

Giannakis, Georgios

Giannakis, Georgios

Gilbert, Keith.

Giri, Ritwik .

Girnyk, Maksym.

Glenn-Anderson, James.

Goeckel, Dennis

Gogineni, Sandeep.

Golato, Andrew...

Goldsmith, Andrea

Goldsmith, Andrea

Golibagh Mahyari, Arash

Gong, Chen

Gong, Chen

Gong, Qipeng

Gonzalez, Gustavo.

Gonzalez Coma, Jose Pablo

Goparaju, Sreechakra

Gorsevski, Peter.
SESSION

.TA4b-1

TA8a3-3

.. TA3a-3

TP8a3-5

MP8a1-2

.. TP6b-2

TA8a2-7

.. TA7b-1

..MP6a-1

TA8b3-1

TA8b3-2

TA8a3-8

.MP7a-1

.. TA1a-1

MP7a-3

MP8a1-7

.. TA1a-3

..MP4b-4

MP6b-1

WA2b-3

MP7a-2

.. TA6b-1

TA8a4-7

MP8a1-6

.MP4b-2

. TA4b-1

.. TA3a-1

..MA5b-3

WA2b-2

TP7b-4

TA8b3-6

TP8a3-6

.. TP8a3-8

..MA1b-4

..MP5a-3

.. TA1b-3

.. TA1b-4

.MP8a3-4

TP3b-3

TP8a1-3

.. TP8b3-7

WA4a-4

TP6a-1

TA7b-4

MP5a-2

.TA6b-4

WA5a-1

MA8b1-6

TP4a-3

TP5a-1

MA8b2-6

TA8b1-1

WA5b-2

MP8a5-3
NAME

SESSION

Grahn, Håkan TP6b-3

Grant, Steven L. .......................... TP2b-1

Grant, Steven L. .......................... TP2b-3

Gregorio, Fernando ....................MA8b2-6

Grenard, Jerry ..............................TA1b-1

Grgicak, Catherine.......................MP8a2-7

Grover, Pulkit..............................MP1a-3

Gründinger, Andreas...................TA8b1-1

Gu, Erdan .............................. TP7b-2

Gu, Renliang.............................. TP3b-4

Gu, Yi......................................MP8a1-7

Guerra, Anna .............................MP6b-2

Guicquero, William ......................TA8a2-5

Guidi, Francesco .........................MP6b-2

Gunther, Jacob H........................ WA3a-2

Gunther, Jacob H........................ WA3b-1

Guo, Jun .................................MP8a5-6

Gurakan, B. ............................. TP4b-3

Gurbuz, Ali Cafer .........................MP3b-3

Gurbuz, Sevgi Zubeyde...............MP8a4-7

Haardt, Martin ............................MP1b-3

Haardt, Martin ............................ WA4a-3

Haas, Harald............................... TP7b-2

Hague, David ............................MA8b3-2

Haimovich, Alexander...................MP6b-1

Haimovich, Alexander................... TA6a-1

Hajibabaei, Zahra ........................ WA6b-3

Hakhamaneshi, Farhood .............MP8a4-6

Hall, Eric ........................................TA2a-3

Han, Keyong..................................MP6a-2

Hannig, Frank ..............................MP7a-3

Hanrahan, Sara ..........................MA8b4-7

Hansen, Martin Weiss .................MP8a5-4

Hansson-Sandsten, Maria ...........TA8b4-8

Hao, Jun ................................. TP8b4-1

Harada, Noboru ........................... TA5a-3

Harati, Amir ................................MP2a-2

Harms, Andrew ............................. TA6a-2

Hassan, Yahia ...........................MP8a4-8

Haubelt, Christian ..........................MP7a-2

Havlicek, Joseph .........................MA5b-2

Hayat, Majeed ............................MA8b3-8

Heath Jr., Robert W. .......................TA4a-3

Heath Jr., Robert W. .....................TA8a1-1

Heath Jr., Robert W. .................. TP8b2-5

Heath Jr., Robert W. .................. WA1a-3

Hebb, Adam ...............................MA8b4-7

Hegde, Rajesh M........................MP8a5-1

Hegde, Rajesh M.........................TA5a-1

Hegde, Rajesh M...................... TP8a4-7

Hellings, Christoph .....................MA8b2-2

Henney, Carl.................................MA5b-4

Himed, Braham........................... TP6a-2

Hindborg, Andreas....................... TP7a-2 


\section{NAME}

Ho, Chung-Cheng

Ho, Matthew

Hochwald, Bertrand

Hock, Rachel

Honrao, Bhagyashri

Hormigo, Javier

Hotz, Thomas

Hsu, Yu-Chang

Hua, Yingbo

Huang, Boyang

Huang, Chu-Hsiang

Huang, Howard.

Huang, Kaibin .

Huang, Lei ....

Huang, Yi...

HudachekBuswell, Mary.

Huemer, Mario

Hui, Dennis

Hwang, Jeng-Kuang

Hwang, Jeng-Kuang

Hwang, Suk-seung

Hwang, Suk-seung

Hyun, Inha

Hyun, Inha.

Ibars, Christian

ljaz, Muhammad

Inan, Huseyin Atahan

Ingle, Atul..

Ingle, Atul.

Iqbal, Naveed

J. Thiagarajan, Jayaraman ..

Jacob, Mathews.

Jafarkhani, Hamid

Jaffard, Stephane.

Jahja, Rico.

Jain, Akshay

Jain, Ayush

Jakobsson, Andreas

Jakobsson, Andreas

Jalali, Ali.

Jalali, Bahram.

Jamalabdollahi, Mohsen

Jamali, Mohsin M.

Jamali, Mohsin M.

Janda, Carsten Rudolf.

Janneck, Jörn W.

Janneck, Jörn W.

Janneck, Jörn W.

Jaouen, Yves

Jarrah, Amin

Jatla, Venkatesh

Jelili, Adebello.

Jensen, Jesper Rindom

Jensen, Jesper Rindom
SESSION

.MP6a-4

TA8b1-6

...TA3b-4

..MA5b-4

WA1b-3

.TA7a-4

TA8b4-5

.TA8a3-7

TP5b-2

TP4a-3

WA4b-1

WA7a-3

TP4b-4

.TA1a-3

..MP4a-1

TP8a3-3

TA8a4-1

MA8b2-3

TA8a1-4

TA8a3-7

MA8b1-2

TA8b3-3

MA8b2-5

TP8b2-8

TP8b1-7

... TP7b-2

..MP8a3-2

MP8a2-5

...TA5b-2

TA8a3-6

TP3a-1

MP3b-4

WA6b-1

...TA5b-4

MA8b1-2

TA8b3-8

.MP8a5-1

TA8b4-4

TA8b4-8

TP1a-4

TA8a2-3

.MP4a-3

MP8a5-3

TP8b3-3

WA4a-2

TP7a-1

TP8b3-4

TP8b3-5

TP4a-1

. TP8b3-3

...MA5b-4

.MA8b3-8

MP8a5-4

TP5a-3
NAME

Jerbi, Khaled

SESSION

Jia, Chao

WA7b-1

Jiang, Feng

TA1a-2

Jiang, Huaiguang

MP8a1-7

Jiang, Huaiguang.

TA1a-3

Jiang, Huiling

WA2a-3

Jo, Sun

TP8b2-8

Joham, Michael

TA8b1-1

Johansen, Christopher ...................TA1b-1

Johansson, Mikael.

TA3a-1

Johnson, Ben.

TA7b-1

Johnson, Christopher ................... TP1a-4

Johnson, Jamie

WA5a-3

Johnson, Richard

TA5b-1

Jones, Aaron

WA6a-1

Jorswieck, Eduard A.

TA8a3-1

Jorswieck, Eduard $A$.

TP8b2-2

Jorswieck, Eduard A.

WA4a-2

Jun, Kihwan

TA8b2-1

Kabal, Peter

TP5a-1

Kailkhura, Bhavya

MA4b-1

Kamamoto, Yutaka

TA5a-3

Kang, Jaewook.

TP8a2-2

Kar, Soummya

MP7b-2

Kar, Soummya

TA6b-3

Kar, Soummya

TP1b-2

Karakonstantis, Georgios ...........MP8a4-2

Karakonstantis, Georgios ........... TP8b1-5

Karlsson, Marcus...........................MP4b-3

Karlsson, Sven …........................... TP7a-2

Karnick, Harish

TA5a-1

Karypis, George

.MP1b-1

Kassam, Saleem

TP8a2-1

Katz, Eyal

TP8b1-3

Kayama, Hidetoshi

WA2a-3

Kaynak, Unver

MP8a4-7

Keilholz, Shella.

MP8a2-4

Kekatos, Vassilis

MA1b-4

Kekatos, Vassilis

TP3a-2

Keller, Catherine

TP3a-4

Keogh, Eamonn

MP7a-4

Khan, Usman A.

TP8a4-3

Khan, Usman A.

TP8a4-4

Khan, Usman A

WA5a-4

Khayambashi, Misagh

MP3b-1

Kiah, Han Mao

WA5b-1

Kiah, Han Mao

WA5b-2

Kim, Changkyu

Kim, Haley

MP8a1-2

Kim, Hyun.

TA6a-1

Kim, Jinsub . _...MP5a-1

Kim, Kiseon …............................ TP8a2-2

Kim, Minji ..................................... TP2a-3

Kim, Seung-Jun ..............................MP5a-3 


\section{NAME}

Kim, Seung-Jun

Kim, Sungo

Kirilmaz, Tunahan

Kirsteins, Ivars

Klausmeyer, Philip

Klein, Andrew G.

Klein, Andrew G.

Knopp, Raymond

Ko, Youngwook

Koivunen, Visa.

Koivunen, Visa

Korpi, Dani.

Kose, Abdulkadir.

Kothandaraman, Premnishanth.... TP3a-1

Kovvali, Narayan

Krc, Tomas.

Krishnamurthy, Akshay

Krishnamurthy, Ram

Kroger, Jim

Kronvall, Ted

Kruger, Anton

Krzymien, Lukasz

Krzymien, Witold

Kuehn, Volker

Kuhn, Marc

Kulkarni, Mandar

Kumar, P. R.

Kumar, Santosh.

Kumar, Sudhir.

Kundu, Debarati.

Kupriianova, Olga

Kurkoski, Brian

Kurras, Martin.

Kwon, Goo-Rak .

Kwon, Goo-Rak .

Labeau, Fabrice.

Lai, Lifeng

Lai, Lifeng

Laiw, S K.

Lakshmi Narasimhan, Theagarajan

Lam, Tu Thanh

Lameiro, Cristian

Lang, Oliver

Lanterman, Aaron

Lao, Yingjie.

Lari, Vahid

Larsson, Erik G...

Lashkari, Khosrow

Laubichler, Manfred

Lauter, Christoph ..

Lavrenko, Anastasia

Lawlor, Sean.

Learned, Rachel
TA3b-3

SESSION

. TP8a2-6

MA8b2-5

.MP8a4-7

TP8b1-8

TA5b-3

.TA5b-3

TP8a4-8

TA8b1-3

WA4a-1

..MA1b-2

TP8a4-5

TA8a1-5

TA8a4-8

MA8b4-7

..TA5b-2

...MP3a-3

...TA7a-1

.MA8b4-2

TA8b4-4

TA8b4-1

WA1a-4

TP8b2-7

TP8a2-4

TA8b1-5

WA1a-1

..TA6b-2

WA2b-1

TP8a4-7

TA8a2-8

.TA8b2-8

TA8a3-5

.TA8b1-2

MA8b1-2

TA8b3-3

TP3a-3

MA4b-4

MP4a-1

TP7b-4

TP8a1-8

..TA4b-3

TA8a4-1

MA8b3-5

TA8b2-6

MP7a-3

MP4b-3

TA8a4-5

WA5a-2

TA8b2-8

TA8b4-5

MP7b-1

TA8b1-6

\section{NAME}

Lee, Chung Ghiu

SESSION

Lee, Donghoon

TP7b-4

Lee, Gwo Giun (Chris).................. WA7b-3

Lee, Heung-No ............................. TP8a2-2

Lee, Hyuk-Jae ................................ WA7b-2

Lee, Kanghee ...............................MA8b2-5

Lee, Kanghee .............................. TP8b2-8

Lee, Meng-Ying .............................WA1b-2

LeMinh, Hoa ................................. TP7b-4

Leonardi, Nora ................................MP2b-4

Lerman, G....................................MA1b-3

Leus, Geert .................................MA3b-3

Leus, Geert.....................................TA4a-3

Leus, Geert................................. TP3b-1

Leus, Geert..................................WA6b-2

Lev-Ari, Hanoch .............................MP8a4-5

Lherbier, Regis ............................TA8a2-7

$\mathrm{Li}$, Bo-Syun .................................. WA7b-3

$\mathrm{Li}$, Hongbin ................................... TP6a-2

Li, Jeng-Da ................................. TA8a3-7

$\mathrm{Li}$, Jian .......................................TA7b-3

Li, Jian ......................................TA8b3-8

$\mathrm{Li}$, Jichuan .................................MP8a1-5

$\mathrm{Li}$, Juane ......................................MA7b-1

Li, Kaipeng....................................WA7a-4

Li, Min .......................................MA8b2-1

Li, Minyue ......................................MP8a5-6

$\mathrm{Li}$, Shang-Bin ............................. TP4a-2

Li, Shuo …..................................MP8a3-3

$\mathrm{Li}$, Ting .........................................

Li, Xin.........................................MP5b-3

$\mathrm{Li}$, Yang........................................TA4a-1

Li, Yao ......................................... WA4b-1

Li, Yun........................................MP1a-1

Lian, Jie ................................... TP7b-1

Liang, Yingbin ...............................MA4b-4

Lin, Chuan-Shun ...........................TA8a1-4

Lin, Chuan-Shun............................TA8a3-7

Lin, Min .....................................MA8b2-1

Lin, Min .................................... TP8b2-3

Lin, Pin-Hsun .............................. TA8a3-1

Lin, Shu ........................................

Lin, Xuehong ...............................A8b3-3

Lin, Yuan-Pei ....................................8b1-4

Little, Thomas ................................ TP7b-3

Liu, Bin...................................... TP8b3-2

Liu, Brian ........................................A8b4-3

Liu, Chun-Lin ...............................MP8a3-5

Liu, Jen-HaO ...............................TA8a1-4

Liu, Keke......................................MA7b-1

Liu, Weigang.............................. TP8a4-2

Liu, Weihao................................. TP8b1-2

Lops, Marco ..................................MP6b-1

Love, David.....................................TA3b-2 


\begin{tabular}{|c|c|}
\hline $\begin{array}{r}\text { SESSION } \\
\ldots \ldots . . \text { TA8a1-6 }\end{array}$ & $\begin{array}{l}\text { NAME } \\
\text { Melzer, Jordan ....... }\end{array}$ \\
\hline Low, Steven...... & Memarian, Negar... \\
\hline Lozano, Angel.................. & Messier, Paul .............. \\
\hline Lu, Lei.................................. & Mikhael, Wasfy ........... \\
\hline Lu, Yue .......................................... TP8b4 & Milenkovic, Olgica............ \\
\hline Lu, Yue M. . & Milenkovic, Olgica... \\
\hline utz, David... & Milenkovic, Olgica...... \\
\hline ... TP8a3-5 & Minot, Ariana................. \\
\hline Ma, Shuoxin...... & Mirkin, Mitch .... \\
\hline Ma, Xiaoli....... & Mirza, Usman Mazhar ...... \\
\hline Ma, Zhanyu...... & Mirzaei, Golrokh .............. \\
\hline Maalouli, Ghassan....... & Mishra, Kumar Vijay ........ \\
\hline Macagnano, Davide............. & Miyabe, Shigeki ................... \\
\hline Madhow, Upamanyu............. & Mo, Jianhua ........................ \\
\hline Madhow, Upamanyu..... & Moallemi, Nasim..... \\
\hline Magnússon, Sindri....... & Mogensen, Preben ......... \\
\hline Mahajan, Divya................... & Moinuddin, Mohammad.... \\
\hline Maharaj, Sunil (B.T.).. & Mokhtari, Aryan.................. \\
\hline Mahmood, Mir H..... & Mollison, Matthew... \\
\hline Mahoor, Mohammad... & Mönich, Ullrich........... \\
\hline Mahzoon, Majid .............. & Mookherjee, Soumak...... \\
\hline Makino, Shoji................... & Moon, Changki ............... \\
\hline Malekzadeh, Masoud. & Moon, Changki ........... \\
\hline Malysa, Greg ................ & Moon, Sunghoon ... \\
\hline Mamandipoor, Babak....................MP8a4-4 & Moon, Sunghoon ........................ TP8b2-8 \\
\hline Manduca, Armando .....................MP8a2-3 & Moon, Todd K. ............................. \\
\hline Mansukhani, Jyoti.............. & Moon, Todd K..... \\
\hline Manzoor Siddiqui, Fahad.. & Moore, Linda...... \\
\hline Mardani, Davood ............... & Moreau, Eric ........... \\
\hline Mardani, Morteza..... & Moriya, Takehiro .......... \\
\hline Maric, Ivana ............ & Morsi, Rania ........... \\
\hline Markovic, Dejan... & Moulin, Pierre ..................... \\
\hline Marlow, Ryan................ & Mudumbai, Raghuraman .... \\
\hline Marot, Julien ....................... & Mukherjee, Amitav .........................MP8a1-4 \\
\hline Marshall, Alan ..................... & Mungara, Ratheesh ................... TP8b2-5 \\
\hline Martin, Rainer ....................... & Musaddiq, Matheen ......................TA8b2-5 \\
\hline Masazade, Engin .................. & Nachiappan, Ramanathan ............. TP3a-1 \\
\hline Mathew, Sanu......................... & Nadakuditi, Rajesh .........................TA1a-4 \\
\hline Mattavelli, Marco ................... & Nafie, Mohammed ............. \\
\hline Matthiesen, Bho.................. & Nam, Young-Han ................. \\
\hline Maurandi, Victor . & Naqvi, Syed Hassan Raza............TA8b1- \\
\hline Maurer, Alexander ........ & Naseri, Hassan ............................ TP8a4-5 \\
\hline McClure, Neil............. & Naskovska, Kristina ........... \\
\hline McEachen, John.................... & Nassif, Roula ................................. \\
\hline McKay, Matthew .................. & Natesan Ramamurthy, Karthikeyan... \\
\hline McKendry, Jonathan J. D. . & \\
\hline McRae, Nathan....................... & Nathwani, Karan ...........................MP8a5-1 \\
\hline McWhirter, John....... & Navab, Nassir ...................... \\
\hline Médard, Muriel.......... & Navarro, Monica ....................... TP8b1-7 \\
\hline Medda, Alessio ...... & Navasca, Carmeliza ........................TA1a-1 \\
\hline Medda, Alessio ...... & Nayar, Himanshu ............................ TA1a-4 \\
\hline Mehanna, Omar.... & Needell, Deanna............................. 1 1b-1 \\
\hline Melodia, Tommaso & Needell, Deanna......................... TP8a3-5 \\
\hline 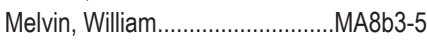 & Nehorai, Arye...... \\
\hline
\end{tabular}




\section{NAME}

Nehorai, Arye

Nehorai, Arye

Nema, Shikha

$\mathrm{Ng}$, Derrick Wing Kwan

Nguyen, Chuong

Nguyen, Dang Khoa

Nguyen, Lam

Nguyen, Lam

Nguyen, PhuongBang

Nie, Ding...

Nieh, Jo-Yen

Nitinawarat, Sirin

Niu, Zhisheng

Noh, Eunho.

Nokleby, Matthew

Nordström, Tomas

Norman, Mark.

Noshad, Mohammad.

Noubir, Guevara

Noujeim, Karam.

Nourani, Mehrdad.

Noyer, Jea-Charles.

Obeid, lyad

Ochi, Hiroshi.

Ogunfunmi, Tokunbo ....................... TA5a-2

Ojowu, Ode.

Okopal, Greg

Oliveras Martinez, Alex.

Ollila, Esa

Olofsson, Andreas

Olorode, Oluleye...

Orhan, Umut.

Oshiga, Omotayo

Otazo, Ricardo

Ouyang, Jian

Oweiss, Karim

Ozdemir, Alp

Ozel, 0 .

Ozer, Sedat.

Pacheco, Courtney.

Padaki, Aditya.

Pados, Dimitris A.

Pakrooh, Pooria.

Pal, Piya

Paleologu, Constantin

Palka, Thomas.

Palomar, Daniel .

Palomar, Daniel .

Pan, Yen-Chang

Papandreou-Suppappola, Antonia.

Papandreou-Suppappola, Antonia...

Parhi, Keshab K
MA8b4-7

WA6a-3

TP8a1-8

...

..TA3b-4

TP8a2-5

MP1a-1

WA2a-1

MA2b-3

TA8a3-5

TP6b-4

.MA8b4-1

TP7b-1

...TA8a3-3

.MP8a4-4

TP8b3-6

.TA8a2-7

MP2a-2

TP8a1-8

..TA7b-3

TP5a-4

TA8a3-8

MA1b-2

TP6b-4

TP8b3-6

MA2b-4

.. TP8a4-1

.MP8a2-6

.. TP8b2-3

..TA2a-4

.MP8a5-5

TP4b-3

MA5b-1

MA2b-1

TP8a1-4

.. TP8a1-6

MA6b-1

MA6b-4

TP2b-3

..MP8a3-6

TP3b-2

TP8b2-4

MA8b1-4

MP8a4-3
NAME

Parhi, Keshab K

SESSION

Parhi, Keshab K........................ TP8b4-5

Parhi, Keshab K ........................... WA7a-2

Parhi, Megha ..............................TA8b2-6

Paris, Alan ..................................MA8b4-6

Parker, Thomas ............................ WA5a-3

Parkvall, Stefan ..............................TA4a-2

Parvania, Masood..........................TA6b-1

Patole, Sujeet ................................WA1b-1

Pattichis, Marios ...........................MA5b-2

Pattichis, Marios ...........................MA5b-4

Paul, Bryan .................................. WA6a-3

Payton, Karen...............................MP8a3-4

Peizerat, Arnaud ..........................TA8a2-5

Peng, Yan-Tsung ........................TA8a2-2

Penno, Robert .............................. WA6a-2

Pequito, Sergio .................................TA6b-3

Percus, Allon............................... TP8a3-5

Pereira da Costa, Mario............. TP8a4-5

Pesavento, Marius....................... TP8b2-4

Petersson, Stefan .......................... TP6b-3

Petropulu, Athina ...........................MP3a-2

Pezeshki, Ali ..............................MA6b-1

Pezeshki, Ali .................................MP3a-4

Pfletschinger, Stephan.................. TP8b1-7

Phelps, Shean ............................MA8b4-3

Phoong, See-May.........................MA8b1-4

Picard, David ...............................TA8a2-1

Picone, Joseph ...............................MP2a-2

Pimentel, Jon ............................... TP8b3-1

Pishdad, Leila ............................... TP3a-3

Pishro-nik, Hossein....................... WA4a-4

Pitaro, Michael.............................TA8b1-6

Pitton, James.............................. TP8b4-8

Planjery, Shiva .............................MA7b-3

Plishker, William .............................MP7a-1

Poor, H. Vincent..............................MA4b-3

Poor, H. Vincent..............................MP5a-2

Poor, H. Vincent..............................TA6b-4

Popov, Konstantin........................... TP6b-1

Popovski, Petar .............................TA8a3-8

Pradhan, Sajina ...........................TA8b3-3

Pratschner, Stefan ......................TA8a1-7

Probst, Christian W....................... TP7a-2

Proudler, lan .................................MP8a3-8

Proulx, Brian ................................ TA8b3-7

Purmehdi, Hakimeh ..................... TP8b2-7

Pyun, Jae-young..........................MA8b1-2

Pyun, Jae-young...........................TA8b3-3

Qureshi, Tariq .............................. TP6a-3

Rabbat, Michael............................MP7b-1

Rabbat, Michael.............................TA3a-1

Rabbat, Michael.............................TA3a-3

Rabbat, Michael.............................. TP1b-4 


\section{NAME}

Rabideau, Dan

Radha, Hayder

Radha, Hayder

Radha, Hayder

Rahman, Mehnaz

Rajagopal, Sridhar

Rajaram, Siddharth.

Ramakrishna, Sudhir....

Ramamurthy, Karthikeyan

Ramezani, Hamid

Ramírez, David

Ramlall, Rohan

Rangan, Sundeep.

Rangan, Sundeep.

Rangaswamy, Muralidhar

Rangaswamy, Muralidhar

Rangaswamy, Muralidhar

Rangaswamy, Muralidhar

Rani, Ruchi.

Rao, Bhaskar....

Rao, Bhaskar

Rao, Bhaskar

Rao, Bhaskar

Rao, Nikhil ....

Rasmussen, Lars K.

Ratnarajah, Tharmalingam ..

Raulet, Mickaël

Ravikumar, Pradeep.....

Ravindran, Niranjay.

Raviteja, Patchava..

Ray, Priyadip

Recht, Benjamin

Reed, Jeffrey

Ren, Haibao.

Ren, Zhe.

Renzi, Daniele

Repovš, Grega

Reynolds, Daryl ....

Rhee, Chae Eun.

Ribeiro, Alejandro .....

Richard, Cédric

Richiardi, Jonas.

Riedel, Marc

Riederer, Stephen

Riedl, Thomas

Rigling, Brian

Rigling, Brian

Riley, Robert.

Rish, Irina

Ritcey, James

Rocha, Paula

Rocha, Pedro.

Roemer, Florian.

Rohani, Ehsan
SESSION

.. TA7b-2

MP7b-3

TA8a2-6

TP8a3-1

TA8a3-4

WA1a-2

MA2b-1

WA1a-2

TP8b4-7

MA3b-3

WA3a-4

.MA8b1-5

MP8a1-2

MP8a2-6

MA8b3-1

TP6a-1

TP6a-3

WA6a-1

MP8a5-1

MA6b-2

MP8a1-1

TP3b-3

WA3a-1

..MP8a3-1

TP8a1-1

TP8a4-2

WA7b-1

TP1a-4

MP1b-1

..TA3b-3

..TA4b-2

MP3a-1

TP8a1-4

WA2a-4

TP8b2-1

WA7b-1

TA2b-1

MP5b-3

WA7b-2

TP1b-3

..TA3a-2

MP2b-2

TP8b4-5

MP8a2-3

.MA3b-4

WA6a-1

WA6a-2

MA8b3-6

TA2b-2

WA4b-3

. TA6b-3

TA6b-3

TA8b4-5

MA7b-4
NAME

SESSION

Rohani, Ehsan

TA8a3-4

Roivainen, Jussi

TP6b-2

Romero, Ric.

TP8a2-5

Rong, Yu.

TP5b-3

Ross, Jeremy.

MP8a5-3

Rostamian, Majed

MP6b-4

Roth, Christoph.......................... TP8b1-5

Roux, Stephane ........................... TA5b-4

Rüegg, Tim ................................TA8b1-5

Rulikowski, Pawel........................ WA7a-3

Rupp, Markus ..............................TA8a1-7

Rusek, Fredrik ..............................MP4b-4

Ryou, Jongbum .........................MA8b2-5

Ryou, Jongbum ........................ TP8b2-8

Sabharwal, Ashutosh.................... TP5b-4

Saeedi, Ramyar .......................... WA2b-2

Safavi, Sam ............................. TP8a4-3

Sagratella, Simone .......................MA1b-1

Sahu, Anit ....................................MP7b-2

Sala, Frederic ........................... WA5b-3

Salah, Aya ..............................MA8b2-4

Salehi, Masoud ........................... TA8a3-3

Salehi, Sayed Ahmad ................ TP8b4-5

San Antonio, Geoffrey ..................TA7b-1

Sangari, Arash ............................MP8a5-2

Sani, Alireza................................ TP8a2-3

Sankaranarayanan, Preethi.........MP8a2-2

Santamaria, Ignacio.....................TA4b-3

Santamaría, Ignacio .................... WA3a-4

Santhanam, Balu .........................MA8b3-8

Santhanam, Sridhar......................TA7b-4

Sarayanibafghi, Omid ..................TA8b4-7

Sarkar, Rituparna..........................MA5b-1

Sartori, Philippe .......................... WA1a-4

Satpathy, Sudhir ..........................TA7a-1

Sattigeri, Prasanna .................... TP8b4-7

Sayed, Ali H................................ TA3a-2

Sayeed, Akbar ...........................MA8b1-7

Scaglione, Anna........................... TA3a-4

Scaglione, Anna........................... TA6b-1

Scaglione, Anna.......................... TP8a1-2

Schaefer, Rafael F. ......................MA4b-3

Scharf, Louis L.............................MA6b-1

Scharf, Louis L............................. WA3a-4

Scheunert, Christian ..................... WA4a-2

Schizas, loannis ......................... TP1b-1

Schleuniger, Pascal ...................... TP7a-2

Schniter, Philip............................MA8b1-7

Schniter, Philip............................TA8a1-1

Schober, Robert........................... TP4b-2

Schoeny, Clayton.........................WA5b-3

Schomay, Theodore ...................MP8a2-2

Schreier, Peter J.......................... WA3a-4

Schulte, Michael .......................... TA7a-2 


\section{NAME}

Schupp, Daniel

Schwartz, Moshe

Schwarz, Stefan

Scrofani, James.

Scutari, Gesualdo

Sen Gupta, Ananya

Senay, Seda

Sethares, William.

Sethares, William.

Sethares, William.

Setlur, Pawan

Seto, Koji

Severi, Stefano

Sevuktekin, Noyan.

Shabeeb, Mahdy

Shah, Mohit

Shah, Parikshit

Shah, Parikshit

Shahbazpanahi, Shahram.

Sheikholeslami, Azadeh ...

Sheikholeslami, Fatemeh

Shekaramiz, Mohammad

Shi, Zhijie.

Shin, Seokjoo

Shin, Seokjoo

Shinn-Cunningham, Barbara ........MA2b-1

Shinotsuka, Marie.

Shirazi, Mojtaba.

Shynk, John J.

Sidiropoulos, Nicholas.

Sidiropoulos, Nicholas.

Silva, Vitor

Simonetto, Andrea

Singer, Andrew

Singer, Andrew

Singer, Andrew

Singh, Aarti

Singh, Sarabjot

Sinno, Zeina

Skadron, Kevin

Skeppstedt, Jonas

Sklivanitis, George.

Skoglund, Mikael

Slavakis, Konstantinos

Slavakis, Konstantinos

Smith, Shaden

Song, Junxiao.

Soong, Anthony .

Sørensen, Troels B.

Soury, Hamza

Sousa, Ericles.

Spagnolini, Umberto

Spagnolini, Umberto

Spanias, Andreas
SESSION

TP8b1-8

TP2a-1

TA8a1-7

WA5a-3

MA1b-1

TP8b1-8

. TP8a3-4

MP8a2-5

MP8a5-2

..TA5b-2

MA8b3-1

.TA5a-2

TA8b3-6

. TP8b1-4

TP8b2-1

TP8b4-7

..MP3a-1

MP8a3-1

TP8a3-7

WA4a-4

...TA1b-4

WA3a-2

MP4a-1

MA8b1-2

TA8b3-3

TA8b3-4

.. TP8a2-7

MP8a1-6

..MP1b-1

.TA8a1-8

.MP8a4-2

WA6b-2

...MA3b-4

TP8b1-4

TP8b4-6

MP3a-3

WA1a-1

..MP5b-1

MA5b-1

TP7a-1

TP8a1-6

TP8a1-1

..MA1b-3

...TA1b-3

..MP1b-1

TP3b-2

WA1a-4

MP8a4-6

WA6a-4

MP7a-3

TA8b1-8

TP8b2-6

TP8b4-7
NAME

SESSION

Speranzon, Alberto .......................TA8a4-7

Sridhar, Rahul .............................. TP3a-1

Stanacevic, Milutin........................MP8a3-3

Stanczak, Slawomir ..................... TP8b2-1

Stathakis, Efthymios .................... TP8a1-1

Steinwandt, Jens ...........................WA4a-3

Stewart, Michael ........................... TP8a3-3

Stojanovic, Milica...........................MA3b-3

Stojanovic, Milica...........................MP4a-2

Stroder, Amy ................................ TP8b4-1

Strohmer, Thomas ..........................MP6a-1

Strother, Stephen............................MP2b-1

Struder, Christoph........................ TP8b1-5

Stuijk, Sander …..........................MP7a-2

Su, Borching .................................MP6a-3

Su, Borching ................................. TA8a1-3

Su, Borching ...................................WA1b-2

Su, Lili......................................... WA5b-1

Sulaman, Sardar Muhammad.... TP8b3-4

Sullivan, Michael...........................TA8b2-2

Sun, Longji..................................TA8b3-5

Sun, Shunqiao ...............................MP3a-2

Sun, Wensheng .............................MP4a-3

Suo, Yuanming ..............................MA6b-3

Suppappola, Seth .........................TA8a4-5

Surana, Amit ............................... TA8a4-7

Suresh, Vikram ................................TA7a-1

Swamy, M.N.S. ............................ TP5a-2

Swärd, Johan................................TA8b4-4

Swärd, Johan..................................TA8b4-8

Swartzlander, Earl ........................TA8b2-1

Swartzlander, Earl ........................TA8b2-2

Swartzlander, Earl .......................TA8b2-5

Swenson, Brian ............................. TP1b-2

Swindlehurst, A. Lee......................TA1a-2

Swindlehurst, Lee ..........................MP3b-1

Tajer, Ali ..........................................MP1a-4

Talwar, Shilpa .............................. TP5b-1

Tanan, Subhash...........................MP8a5-1

Tanchuk, Oleg................................MA6b-2

Tandon, Ravi............................... TP8a1-4

Tang, Gongguo .............................MP3a-1

Tang, Ming-Fu...............................WA1b-2

Taori, Rakesh................................. WA1a-2

Tarango, Joseph ............................MP7a-4

Tavares, Fernando M. L...............MP8a4-6

Teich, Juergen ...............................MP7a-3

Teixeira, Andr'e ..............................TA3a-1

Teke, Oguzhan ...............................MP3b-3

Temlyakov, Vladimir ...................... TP1a-3

Tenneti, Srikanth Venkata............ WA3a-3

Theelen, Bart................................MP7a-2

Thiagarajan, Jayaraman ............. TP8b4-7

Thiele, Lars...................................TA8b1-2 


\section{NAME}

Thomae, Reiner.

Thomas, Robert.

Thomas, Robin

Thomas, Timothy

Thompson, Keith

Tonelli, Oscar....

Tong, Lang.....

Toriyama, Yuta

Torlak, Murat

Traganitis, Panagiotis ..

Tran, Trac

Tran, Trac

Tripathy, Abhijit

Trzasko, Joshua

Tsakiris, Manolis

Tseng, Kai-Han

Tsianos, Konstantinos .

Tsonev, Dobroslav ....

Tufvesson, Fredrik.

Tullberg, Hugo

Tummala, Murali

Tyagi, Himanshu

ul-Abdin, Zain

Ulukus, Sennur

Utschick, Wolfgang.

Utschick, Wolfgang.

Utschick, Wolfgang.

Vaccaro, Richard ...

Vaidyanathan, P. P.

Vaidyanathan, P. P.

Vaidyanathan, P. P.

Vaidyanathan, P. P.

Vakili, Sattar......

Valdivia, Nicolas

Valkama, Mikko

Van de Velde, Samuel

Van De Ville, Dimitri.

Vandergheynst, Pierre.

Varghese, Lenny.

Varghese, Tomy.

Varshney, Pramod

Varshney, Pramod

Varshney, Pramod

Vary, Peter.

Vasic, Bane

Vaughan, Andrew ....

Veeravalli, Venugopal

Vehkaperä, Mikko

Venkateswaran, Vijay

Verde, Francesco

Vía, Javier......

Vidal, Rene

Vilà-Valls, Jordi

Villafañe-Delgado, Marisel...........MA8b4-5

.MP5a-1

TA8b1-3

..TA4a-4

MP8a3-8

..MP8a4-6

..MP5a-1

WA7a-1

WA1b-1

. TA1b-3

MA6b-3

. TA6a-4

MP8a5-1

.MP8a2-3

TP1a-1

TA8a1-3

TP1b-4

TP7b-2

MP4b-4

..TA4a-2

WA5a-3

MA4b-2

TP6b-4

TP4b-3

..MA8b2-2

.. TA4b-3

TA8b1-1

.MP8a3-6

WA3a-3

MA6b-4

MP8a3-5

TP8b4-4

WA3b-3

MA8b3-7

TA8a1-5

TA8b3-6

.MP2b-4

TA8a2-5

.MA2b-1

.MP8a2-5

.MA4b-1

TA4b-2

TP6a-4

TP2b-2

MA7b-3

MA8b4-3

MP1a-1

TP8a1-3

WA7a-3

.. TA6b-1

WA3a-4

TP1a-1

MP6b-3
NAME

Villalba, Julio

SESSION

Vook, Frederick.

TA7a-4

Vorobyov, Sergiy

Vorobyov, Sergiy

Vosoughi, Aida

Vosoughi, Azadeh

Vosoughi, Azadeh

Vosoughi, Azadeh

Vosoughi, Azadeh.

.TA4a-4

Vouras, Peter.

TP8a1-3

WA4a-3

Vuppala, Satyar

Wage, Kathleen

Wagner, Kevin

MP8a4-2

MA8b4-6

TP8a2-3

TP8a2-7

WA6b-3

TP8a2-8

TP8a4-2

TP8b3-8

Wai, Hoi To

TA8a4-2

Walter, Maxwell.

TA3a-4

Walters, George

TP7a-2

Wang, Gang

TA8b2-3

Wang, Guohui

MA1b-4

Wang, Guohui.

MP8a4-2

Wang, Rui WA7a-4

Wang, $X$

MP8a1-3

Wang, Xin

MA1b-3

Wang, Yiyin

MA8b3-3

.....MP4a-3

Wang, Zhongfeng ........................MA7b-2

Warty, Chirag ..............................WA1b-3

Wassie, Dereje A. .......................MP8a4-6

Watanabe, Shun ...........................MA4b-2

Weavers, Paul ............................MP8a2-3

Weeraddana, P. Chathuranga ....... TA3a-3

Wei, Ruey-Yi...............................WA4b-3

Wei-Ping, Zhu ............................ TP8b2-3

Weiss, Stephan ..........................MP8a3-8

Wellner, Genevieve ....................MP8a2-7

Wen, Miaowen...............................MP4a-4

Wendt, Herwig .............................. TA5b-4

Wenndt, Stanley .......................... TA5a-4

West, Derek.............................MA8b3-6

Whipple, Gary ............................ TP3a-4

Wijewardhana, Uditha .................TA8b4-6

Wilcher, John ...............................MA8b3-5

Willett, Rebecca...........................TA2a-3

Wimalajeewa, Thakshila...............MA4b-1

Wisdom, Scott ............................. TP5a-4

Wisdom, Scott ............................ TP8b4-8

Wittneben, Armin .......................MP8a4-8

Wittneben, Armin ........................TA8b1-5

Wong, Lok ............................... TP8b1-6

Wood, Sally ...................................TA5b-2

Woods, Damien ........................... TP2a-2

Woods, Roger............................. TP7a-3

Woods, Roger............................ WA4a-1

Wright, Stephen ..........................MP8a3-1

Wu, Dalei................................... TP5a-2 


\section{NAME}

Wu, Michael

Wu, Michael

Wu, Nan

Wu, Qisong

Wu, Qisong

Wu, Yiqun

Wu, Yonglin

Wu, Zhengwei

Xavier, Joao

$\mathrm{Xi}$, Chenguang.

Xi, Peng.

Xia, Xiang-Gen

Xiao, Weimin

Xie, Le

Xu, Jingwei

Xu, Luzhou

Xu, Luzhou

Xu, Tianyi.

Xu, Weiyu

$\mathrm{Xu}$, Weiyu

Xu, Weiyu

Xu, Xiuqiang .....

Xu, Zhengyuan

Xu, Zhengyuan

Xu, Zhengyuan

Xu, Zhengyuan

Xue, Feng

Yamada, Takeshi

Yang, Liuqing....

Yang, Liusha.

Yang, Peng

Yang, Shuo

Yang, Yang

Yen, Chia-Pang

Yener, Aylin

Yin, Bei .

Yin, Bei .

Yin, Haifan

You, Xiaohu

Young, Phillip

Younis, Abdelhamid

Yu, Hong.....

Yuan, Bo

Yuan, Bo

Yuan, Haochen

Yviquel, Hervé

Zaker, Nazanin

Zaki, George.

Zappone, Alessio

Zariffa, Jose
SESSION

MP8a4-1

WA7a-4

TP4a-4

..TA6a-3

TA7b-4

WA2a-2

.MP8a2-7

TP8a2-1

TP1b-2

TP8a4-4

TA8b4-3

..TA3b-1

WA1a-4

..TA6b-2

MA7b-4

..TA7b-3

TA8b3-8

.. TA3b-1

.MP1a-2

TA8a1-2

TA8b4-1

WA2a-2

.MA8b1-6

TP4a-2

TP4a-3

TP8b1-2

TP5b-1

.. TA5a-3

MP4a-4

TP1a-2

MP6a-2

.MA8b3-3

TP8b2-4

WA1b-2

TP4b-1

MP8a4-1

WA4a-1

MP4b-2

MA7b-2

.MP8a2-3

TP7b-2

MP8a5-6

..MP8a4-3

WA7a-2

TA8a2-3

WA7b-1

MA8b4-7

MP7a-1

TA8a3-1

..MP2a-3
NAME

Zekavat, Seyed.

SESSION

Zerguine, Azzedine

MP4a-3

Zerguine, Azzedine

TA8a3-6

Zerguine, Azzedine ....................... TA8a4-6

Zhai, Yixuan................................. TA4b-4

Zhang, Chuan...............................MA7b-2

Zhang, Huishuai ...........................MA4b-4

Zhang, Huishuai ............................MA4b-4

Zhang, Jianshu ............................MP1b-3

Zhang, Jianzhong (Charlie) ........... TA4a-1

Zhang, Jun...................................MA8b4-7

Zhang, Jun.................................... TA1a-3

Zhang, Jun................................. TP8b4-1

Zhang, Jun................................ TP8b4-2

Zhang, Junshan..........................TA8b3-7

Zhang, Mengyi........................... TP8b2-4

Zhang, Shan .............................. WA2a-1

Zhang, Shunqing ......................... WA2a-2

Zhang, Shuo ................................TA8a4-7

Zhang, Xiaoke ............................MA8b1-6

Zhang, Xinchen ......................... TP8b2-5

Zhang, Yimin...............................TA6a-3

Zhang, Yimin............................... TA7b-4

Zhang, Yingchen..........................MP8a1-7

Zhang, Yingchen...........................TA1a-3

Zhang, Yuan ................................MP5b-2

Zhang, Yuanrui ............................. WA4a-1

Zhao, Changhong........................MP5a-4

Zhao, Qing..................................TA4b-4

Zhao, Qing.................................. TP8a1-2

Zhao, Qing.................................WA3b-3

Zhao, Ran......................................TA1b-1

Zhao, Yue ......................................MP5a-2

Zhao, Yue .................................. TA6b-4

Zhou, G. Tong ............................. TA8b3-4

Zhou, Sheng .............................. WA2a-1

Zhou, Shengli ...............................MP4a-1

Zhou, Wentian ............................MP5b-3

Zhou, Yuan ............................... WA3b-3

Zhou, Zhichong........................... TP8b4-2

Zhu, Jinkang ................................ WA2a-4

Zhu, Meifang..............................MP4b-4

Zhu, Wei-Ping ............................MA8b2-1

Zhu, Wei-Ping .............................. TP5a-2

Zoechmann, Erich ....................... TA8a1-7

Zong, Pingping ...........................TA8b1-7

Zorzi, Michele ...............................MA3b-1

Zou, Difan .................................... TP4a-2 


\section{Notes}




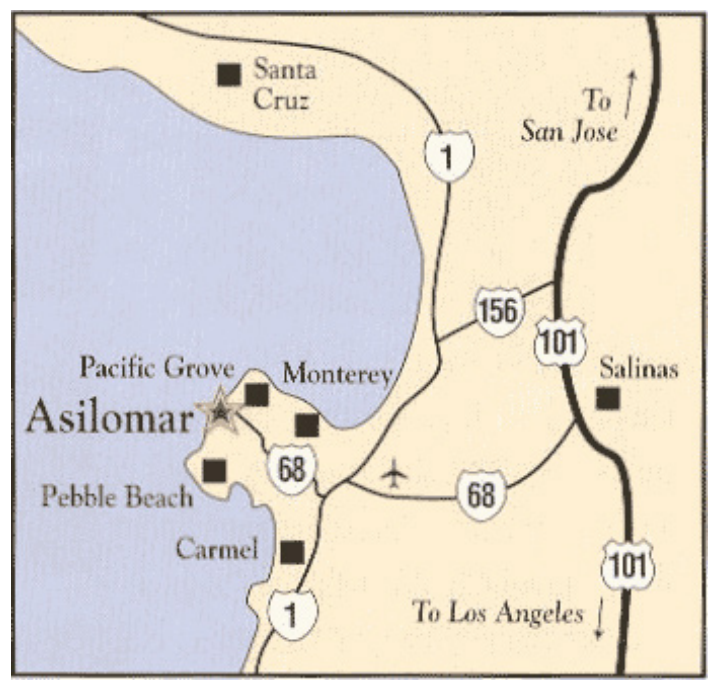




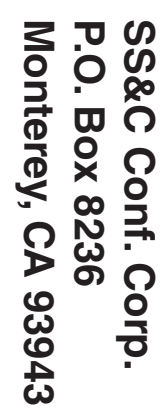

